\title{
Small angle Bhabha scattering at LEP1. Wide-narrow angular acceptance.
}

\author{
N.P.Merenkov \\ National Science Centrd " Kharkov Institute of Physics and Technology" \\ Academicheskaya Str.1, 310108, Kharkov, Ukraine
}

\begin{abstract}
Analytical method is applied for description of the small angle Bhabha scattering at LEP1. Inclusive event selection for asymmetrical wide-narrow circular detectors is considered. The QED correction to the Born cross-section is calculated with leading and next-to-leading accuracy in the second order of perturbation theory and with leading one - in the third order. All contributions in the second order due to photonic radiative corrections and pair production are calculated starting from essential Feynman diagrams. The third order correction is computed by means of electron structure function. Numerical results illustrate the analytical calculsations.
\end{abstract}

PACS 12.15.Lk, 12.20.-m, 12.20.Ds, 13.40.-f

\footnotetext{
${ }^{1} \mathrm{E}-$ mail: kfti@rocket.kharkov.ua
} 


\section{Introduction}

The small angle Bhabha scattering (SABS) process is used to measure the luminosity of electron-positron colliders. At LEP1 an experimental accuracy on the luminosity of $\delta \sigma / \sigma<0.1 \%$ has been reached [1]. However, to obtain the total accuracy, a systematic theoretical error must also be added. The accurate determination the SABS cross-section, therefore, directly affects some physical values measured at LEP1 experiments [2,3]. That is why in recent years a considerable attention has been devoted to the Bhabha process [3-11]. The reached accuracy is, however, still inadequate. According to these evaluations the theoretical estimates are still incomplete.

The theoretical calculation of SABS cross-section at LEP1 has to cope with two somewhat different problems. The first one is the description of an experimental restrictions used for event selection in terms of final particles phase space. The second concludes in the writing of matrix element squared with required accuracy. There are two approaches for theoretical investigation of SABS at LEP1: the approach based on Monte Carlo calculation [3-5,7] and semi-analytical one $[6,9-11]$.

The advantage of Monte Carlo method is the possibility to model different types of detectors and event selection [3]. But at this approach one can not use in a strightforward way the exact matrix element squared based on essential Feynman diagrams because of infrared divergence. Therefore, some additinal procedures (YFS factor exponentiation [12], utilization of the electron structure functions [13]) apply to get rid this problem and to take into account leading contribution in the higher orders. It needs to be carefully at this point because of a possibility of the double counting. Any way, up to now the next-to-leading second order correction remains uncertain, and this is transparent defect of Monte Carlo approach.

The advantage of analytical method is the possibility to use the exact matrix element squared. The infrared problem in the frame of this approach is solved by usual way taking into account virtual, real soft and hard photon emission as well as pair production in every order of perturbation theory. Any questions with double counting do not arise at analytical calculations. The defect of this method is its low mobility relative the change of an experimental conditions for event selection. Nevertheless, the analytical calculations have a great importance because allow to check numerous Monte Carlo calculations for different "ideal" detectors. 
Up to now analytical formulae for SABS cross-section at LEP1 are published for the case of inclusive event selection (IES) when circular symmetrical detectors record only final electron and positron energies $[10,11]$. These define the first and second order corrections to Born cross-section with leading (of the order $(\alpha L)^{n}$ ) and next-to-leading (of the order $\alpha^{n} L^{n-1}$ ) accuracy as well as third order one with leading only. Just these contributions will have to be computed to reach required per mille accuracy for SABS cross-section at LEP1. Note that such accuracy selects only collinear (like two-jets final-state configuration) and semicollinear (like three-jets one) kinematics.

In this paper I list full analytical calculation for IES with wide-narrow angular acceptance. The first and second order corrections are derived with next-to-leading accuracy starting from Feynman diagrams for two-loops elastic electron-positron scattering, one-loop single photon emission, two photon emission and pair production. The third order one is obtained with leading accuracy by the help of the electron structure function method. The results for leading second and third order corrections in the case of CES are given too.

The contents of this paper can be outlined as follows. In Section 2 the "observable" crosssection $\sigma_{\text {exp }}$ is introdcued with cuts on angles and energies taken into account, and the first order correction is obtained. In Section 3 the second order corrections are investigated. These include the contributions of the processes of pair (real and virtual) production considered in Subsection 3.1 and two photons (as well real and virtual) emission. In Subsection 3.2 the correction due to one-side two photon emission is considered and in Subsection 3.3 - due to opposite-side one. The expression for the second order photonic correction is given in leading approximation only, while the next-to-leading conribution to it is written in Appendix A for both symmetrical and wide-narrow detectors. The latter does not contain auxiliary infrared parameter. In Section 4 the full leading third order correction is derived using the expansion of electron structure functions. In Section 5 the numerical results suitable for IES are presented. The correspondence of obtained results with another semi-analytical ones is dicussed in Conclusion. In Appendix B some relations are given which have been used in the process of analytical calculations and which will be very useful for numerical ones. 


\section{First order correcion}

Let us introduce dimentionless quantity

$$
\Sigma=\frac{1}{4 \pi \alpha^{2}} Q_{1}^{2} \sigma_{\exp }
$$

where $Q_{1}^{2}=\epsilon^{2} \theta_{1}^{2}$ ( $\epsilon$ is the beam energy and $\theta_{1}$ is the minimal angle of the wide detector). The "experimetally" measurable cross section $\sigma_{\text {exp }}$ is defined as follows

$$
\sigma_{\text {exp }}=\int d x_{1} d x_{2} \Theta d^{2} q_{1}^{\perp} d^{2} q_{2}^{\perp} \Theta_{1}^{c} \Theta_{2}^{c} \frac{d \sigma\left(e^{+}+e^{-} \rightarrow e^{+}+e^{-}+X\right)}{d x_{1} d x_{2} d^{2} q_{1}^{\perp} d^{2} q_{2}^{\perp}},
$$

where $\mathrm{X}$ is undetected final particles, $x_{1}\left(x_{2}\right), q_{1}^{\perp},\left(q_{2}^{\perp}\right)$ are the energy fraction and the transverse component of the momentum of the electron (positron) in the final state. Functions $\Theta_{i}^{c}$ do take into account angular cuts while function $\Theta$ - cutoff on invariant mass of detected electron and positron:

$$
\begin{gathered}
\Theta_{1}^{c}=\theta\left(\theta_{3}-\theta_{-}\right) \theta\left(\theta_{-}-\theta_{1}\right), \Theta_{2}^{c}=\theta\left(\theta_{4}-\theta_{+}\right) \theta\left(\theta_{+}-\theta_{2}\right), \Theta=\theta\left(x_{1} x_{2}-x_{c}\right), \\
\theta_{-}=\frac{\left|\vec{q}_{1}^{\perp}\right|}{x_{1} \epsilon}, \quad \theta_{+}=\frac{\left|\vec{q}_{2}^{\perp}\right|}{x_{2} \epsilon} .
\end{gathered}
$$

In the case of symmetrical angular acceptance

$$
\theta_{2}=\theta_{1}, \theta_{3}=\theta_{4}, \quad \rho=\frac{\theta_{3}}{\theta_{1}}>1
$$

but for wide-narrow one

$$
\theta_{3}>\theta_{4}>\theta_{2}>\theta_{1}, \quad \rho_{i}=\frac{\theta_{i}}{\theta_{1}}>1
$$

Fof numerical calculation ones usually take

$$
\theta_{1}=0.024, \theta_{3}=0.058, \theta_{2}=0.024+\frac{0.017}{8}, \theta_{4}=0.058-\frac{0.017}{8}
$$

The first order correction $\Sigma_{1}$ includes the contributions of virtual and real soft and hard photon emission processes

$$
\Sigma_{1}=\Sigma_{V+S}+\Sigma^{H}+\Sigma_{H}
$$

The contribution due to virtual and real soft photon (with the energy less than $\Delta \epsilon, \Delta \ll 1$ ) may be written as follows ( in this case $x_{1}=x_{2}=1, \quad \vec{q}_{1}^{\perp}+\vec{q}_{2}^{\perp}=0$ )

$$
\Sigma_{V+S}=2 \frac{\alpha}{\pi} \int_{\rho_{2}^{2}}^{\rho_{4}^{2}} \frac{d z}{z^{2}}\left[2(L-1) \ln \Delta+\frac{3}{2} L-2\right], \quad L=\ln \frac{\epsilon^{2} \theta_{1}^{2} z}{m^{2}},
$$


where $z=\left(\vec{q}_{2}^{\perp}\right)^{2} / Q_{1}^{2}$ and $m$ is electron mass.

The second term in r.h.s. of Eq.(4) represents the correction due to hard photon emission by the electron. In this case

$$
X=\gamma\left(1-x_{1}, \vec{k}^{\perp}\right), \quad x_{2}=1, \quad \vec{k}^{\perp}+\vec{q}_{1}^{\perp}+\vec{q}_{2}^{\perp}=0, \quad x_{c}<x_{1}<1-\Delta
$$

It can be derived by integration of the differential cross section of single photon emission over the region

$$
\rho_{2}^{2}<z<\rho_{4}^{2}, \quad x^{2}<z_{1}=\frac{\vec{q}_{1}^{\perp 2}}{Q_{1}^{2}}<x^{2} \rho_{3}^{2}, \quad-1<\cos \varphi<1,
$$

where $\varphi$ is the angle between vectors $\vec{q}_{1}^{\perp}$ and $\vec{q}_{2}^{\perp}$, in the same way as it has been done in [10] for symmetrical angular acceptance. But at this passage I would like to indicate the principle moments of method used largely to obtain the results of the Section 3 and based on the separate calculation of the contributions due to collinear kinematics and semi-collinear one [14].

In collinear kinematics emitted photon moves inside the cone within polar angles $\theta_{\gamma}<\theta_{0} \ll 1$ centred along electron momentum direction (initial: $\vec{k} \| \vec{p}_{1}$ or final: $\vec{k} \| \vec{q}_{1}$ ). In semicollinear region photon moves outside this cones. Because such distinction no longer has physical meaning, the dependence on auxiliary parameter $\theta_{0}$ disappeares in total contribution. This is valid for IES as well as for CES.

Inside collinear kinematics it needs to keep electron mass in differential cross section

$$
\begin{gathered}
d \sigma=\frac{2 \alpha^{3} s}{\pi^{2} q^{2}}\left[\frac{1+x^{2}}{s_{1} t_{1}}-\frac{2 m^{2}}{q^{2}}\left(\frac{1}{s_{1}^{2}}+\frac{x^{2}}{t_{1}^{2}}\right)\right] d \Gamma, \\
d \Gamma=\frac{d^{3} q_{1} d^{3} q_{2} d^{3} k}{\epsilon_{1} \omega 2 \epsilon} \delta^{(4)}\left(p_{1}+p_{2}-k-q_{1}-q_{2}\right),
\end{gathered}
$$

where $q=p_{1}-k-q_{1}, s_{1}=2\left(k q_{1}\right), t_{1}=2\left(k p_{1}\right), s=\left(2 p_{1} p_{2}\right)$ and $p_{1}\left(p_{2}\right)$ is 4-momentum of initial electron (positron). If photon moves inside initial electron cone

$$
\begin{gathered}
s_{1}=x(1-x) \epsilon^{2} \theta_{-}^{2}, t_{1}=-m^{2}(1-x)(1+\eta), q^{2}=-x^{2} \epsilon^{2} \theta_{-}^{2}=-\epsilon^{2} \theta_{+}^{2}, \\
d \Gamma=\frac{m^{2}}{s} \epsilon^{2} \pi^{2} x(1-x) d x d \eta d \theta_{-}^{2}, \quad 0<\eta=\frac{\theta_{\gamma}^{2} \epsilon^{2}}{m^{2}}<\frac{\theta_{0}^{2} \epsilon^{2}}{m^{2}}
\end{gathered}
$$

and one can derive after integration relative $\eta$

$$
\sigma_{\vec{k} \| \vec{p}_{1}}=\frac{2 \alpha^{3}}{Q_{1}^{2}} \int_{\rho_{2}^{2}}^{\rho_{4}^{2}} \frac{d z}{z^{2}} \int_{x_{c}}^{1-\Delta} d x\left[\frac{1+x^{2}}{1-x} \ln \frac{\theta_{0}^{2} \epsilon^{2}}{m^{2}}-\frac{2 x}{1-x}\right] \theta\left(x^{2} \rho_{3}^{2}-z\right) .
$$


The r.h.s.of Eq.(10) corresponds to the contribution of narrow strip with the width $2 \sqrt{z} \lambda(1-x)$ centred around line $z=z_{1}$ in $\left(z, z_{1}\right)$ plane, where $\lambda=\theta_{0} / \theta_{1}$. Really, the condition $\theta_{\gamma}<\theta_{0}$ for initial electron cone may be formulated as follows

$$
\left|\sqrt{z}-\sqrt{z_{1}}\right|<\lambda(1-x), \quad-1<\cos \varphi<-1+\frac{\lambda^{2}(1-x)^{2}-\left(\sqrt{z_{1}}-\sqrt{z}\right)^{2}}{2 \sqrt{z_{1} z}} .
$$

If photon moves inside final electron cone

$$
\begin{gathered}
s_{1}=\frac{1-x}{x} m^{2}(1+\zeta), t_{1}=-(1-x) \epsilon^{2} \theta_{-}^{2}, q^{2}=-\epsilon^{2} \theta_{-}^{2}=-\epsilon^{2} \theta_{+}^{2}, \\
d \Gamma=\frac{m^{2}}{s} \epsilon^{2} \pi^{2} x(1-x) d x d \zeta \frac{d \theta_{-}^{2}}{x^{2}}, 0<\zeta<\frac{\theta_{0}^{2} \epsilon^{2} x^{2}}{m^{2}},
\end{gathered}
$$

and the integration relative $\zeta$ leads to

$$
\sigma_{\vec{k} \| \vec{q}_{1}}=\frac{2 \alpha^{3}}{Q_{1}^{2}} \int_{\rho_{2}^{2}}^{\rho_{4}^{2}} \frac{d z}{z^{2}} \int_{x_{c}}^{1-\Delta} d x\left[\frac{1+x^{2}}{1-x} \ln \frac{\theta_{0}^{2} \epsilon^{2} x^{2}}{m^{2}}-\frac{2 x}{1-x}\right] .
$$

The r.h.s. of Eq.(13) corresponds to the contribution of the strip with the width $2 \sqrt{z} x^{2}(1-x) \lambda$ around line $z_{1}=x^{2} z$ in plane $\left(z_{1}, z\right)$. Really, the condition $\theta_{\gamma}<\theta_{0}$ for final electron cone may be formulated as $|\vec{r}|<\theta_{0}$, where $\vec{r}=\vec{k} / \omega-\vec{q}_{1}^{\perp} / \epsilon_{1}$, and the last reads as

$$
\left|\sqrt{z_{1}}-x \sqrt{z}\right|<x(1-x) \lambda, \quad-1<\cos \varphi<-1+\frac{\lambda^{2} x^{2}(1-x)^{2}-\left(\sqrt{z_{1}}-x \sqrt{z}\right)^{2}}{2 x \sqrt{z z_{1}}} .
$$

Having contributions due to collinear regions now it needs to find the contribution due to semicollinear ones. Supposing $m=0$ in r.h.s. of Eq.(8) the differential cross section suitable for this case may be written as follows

$$
d \sigma=\frac{\alpha^{3} d \varphi d z d z_{1}\left(1+x^{2}\right)}{\pi Q_{1}^{2} z\left(z_{1}-x z\right)}\left[\frac{1}{z_{1}+z+2 \sqrt{z_{1} z} \cos \varphi}-\frac{x}{z_{1}+x^{2} z+2 x \sqrt{z_{1} z} \cos \varphi}\right] d x .
$$

When integrating the first term into the brackets in r.h.s. of Eq.(15) one must use the restriction $\theta_{\gamma}>\theta_{0}$ or

$$
\begin{gathered}
\left|\sqrt{z_{1}}-\sqrt{z}\right|>(1-x) \lambda, \quad-1<\cos \varphi<1 \\
\left|\sqrt{z_{1}}-\sqrt{z}\right|<(1-x) \lambda, \quad 1>\cos \varphi>-1+\frac{\lambda^{2}(1-x)^{2}-\left(\sqrt{z_{1}}-\sqrt{z}\right)^{2}}{2 \sqrt{z z_{1}}}
\end{gathered}
$$

while for the integration the second one - the restriction $|\vec{r}|>\theta_{0}$ or

$$
\left|\sqrt{z_{1}}-x \sqrt{z}\right|>x(1-x) \lambda, \quad-1<\cos \varphi<1
$$




$$
\left|\sqrt{z_{1}}-x \sqrt{z}\right|<x(1-x) \lambda, 1>\cos \varphi>-1+\frac{\lambda^{2} x^{2}(1-x)^{2}-\left(\sqrt{z_{1}}-x \sqrt{z}\right)^{2}}{2 x \sqrt{z z_{1}}} .
$$

The integration (15) over the region (16) leads to

$$
\sigma_{a}=\frac{2 \alpha^{3}}{Q_{1}^{2}} \int_{\rho_{2}^{2}}^{\rho_{4}^{2}} \frac{d z}{z^{2}} \int_{x_{c}}^{1-\Delta} \frac{1+x^{2}}{1-x} d x\left[\left(\ln \frac{z}{\lambda^{2}}+L_{2}\right) \theta_{3}^{(x)}+L_{3} \bar{\theta}_{3}^{(x)}\right] .
$$

Analogous, the integration of r.h.s. of Eq.(15) over the region (17) gives

$$
\sigma_{b}=\frac{2 \alpha^{3}}{Q_{1}^{2}} \int_{\rho_{2}^{2}}^{\rho_{4}^{2}} \frac{d z}{z^{2}} \int_{x_{c}}^{1-\Delta} \frac{1+x^{2}}{1-x} d x\left(\ln \frac{z}{x^{2} \lambda^{2}}+L_{1}\right)
$$

The values $L_{i}$ which enter into Eqs.(18) and (19) are defined as follows

$$
L_{1}=\ln \left|\frac{x^{2}(z-1)\left(\rho_{3}^{2}-z\right)}{(x-z)\left(x \rho_{3}^{2}-z\right)}\right|, \quad L_{2}=\ln \left|\frac{\left(z-x^{2}\right)\left(x^{2} \rho_{3}^{2}-z\right)}{x^{2}(x-z)\left(x \rho_{3}^{2}-z\right)}\right|, \quad L_{3}=\ln \left|\frac{\left(z-x^{2}\right)\left(x \rho_{3}^{2}-z\right)}{(x-z)\left(x^{2} \rho_{3}^{2}-z\right)}\right| .
$$

Beside this the following notations for $\theta$ - functions are used

$$
\theta_{3}^{(x)}=\theta\left(x^{2} \rho_{3}^{2}-z\right), \bar{\theta}_{3}^{(x)}=1-\theta_{3}^{(x)}=\theta\left(z-x^{2} \rho_{3}^{2}\right)
$$

Thus, the $\Sigma^{H}$ may be represented as the sum of (10), (13), (18) and (19) divided by factor $4 \pi \alpha^{2} / Q_{1}^{2}$ or

$$
\begin{gathered}
\Sigma^{H}=\frac{\alpha}{2 \pi} \int_{\rho_{2}^{2}}^{\rho_{4}^{2}} \frac{d z}{z^{2}} \int_{x_{c}}^{1-\Delta} \frac{1+x^{2}}{1-x}\left[\left(1+\theta_{3}^{(x)}\right)(L-1)+K\left(x, z ; \rho_{3}, 1\right)\right] d x, \\
K\left(x, z ; \rho_{3}, 1\right)=\frac{(1-x)^{2}}{1+x^{2}}\left(1+\theta_{3}^{(x)}\right)+L_{1}+\theta_{3}^{(x)} L_{2}+\bar{\theta}_{3}^{(x)} L_{3} .
\end{gathered}
$$

Further I will use the short notations for $\theta$-functions, namely

$$
\theta_{i}^{(x)}=\theta\left(x^{2} \rho_{i}^{2}-z\right), \theta_{i}=\theta\left(\rho_{i}^{2}-z\right), \bar{\theta} i^{(x)}=1-\theta_{i}^{(x)}, \bar{\theta}_{i}=1-\theta_{i}
$$

One may easy to see that $\Sigma^{H}$ for wide-narrow detectors can be derived from $\Sigma^{H}$ for symmetrical ones (see[10]) by the change z-integrations limits

$$
\int_{1}^{\rho^{2}} d z \rightarrow \int_{\rho_{2}^{2}}^{\rho_{4}^{2}} d z
$$

and the substitution $\rho_{3}$ instead of $\rho$ under integral sign.

The third term in r.h.s. of Eq.(4) describes photon emission by the positron. It may be derived by full analogy with $\Sigma^{H}$ except restrictions on variables $z$ and $z_{1}$, namely

$$
1<z<\rho_{3}^{2}, \quad x^{2} \rho_{2}^{2}<z_{1}<x^{2} \rho_{4}^{2}
$$


The contribution of the collinear kinematics $\left(\vec{k} \| \vec{p}_{2}\right.$ and $\left.\vec{k} \| \overrightarrow{q_{2}}\right)$ to single hard photon emission cross section corresponds to the integration over the regions inside strips with width $2 \sqrt{z}(1-x) \lambda$ and $2 \sqrt{z} x^{2}(1-x) \lambda$, respectively. It may be written as follows

$$
\begin{aligned}
& \sigma_{\vec{k}\left\|\vec{p}_{2}, \vec{k}\right\| \vec{q}_{2}}=\frac{2 \alpha^{3}}{Q_{1}^{2}} \int_{1}^{\rho_{3}^{2}} \frac{d z}{z^{2}} \int_{x_{c}}^{1-\Delta} \frac{1+x^{2}}{1-x} d x\left\{\left(\ln \frac{\epsilon^{2} \theta_{0}^{2}}{m^{2}}-\frac{2 x}{1-x}\right) \Delta_{42}^{(x)}+\right. \\
&\left.\left(\ln \frac{\epsilon^{2} \theta_{0}^{2} x^{2}}{m^{2}}-\frac{2 x}{1-x}\right) \Delta_{42}\right\},
\end{aligned}
$$

where

$$
\Delta_{42}^{(x)}=\theta_{4}^{(x)}-\theta_{2}^{(x)}, \quad \Delta_{42}+\theta_{4}-\theta_{2}
$$

The contribution of semi-collinear kinematics may be derived by integration (15), taking into account the restrictions (16), (17) and (22). The latters correspond to regions outside narrow strips near $z_{1}=z$ and $z_{1}=x^{2} z$, respectively. The result is

$$
\begin{gathered}
\sigma_{a}+\sigma_{b}=\frac{2 \alpha^{3}}{Q_{1}^{2}} \int_{1}^{\rho_{3}^{2}} \frac{d z}{z^{2}} \int_{x_{c}}^{1-\Delta} \frac{1+x^{2}}{1-x} d x\left[\ln \frac{z}{\lambda^{2}}\left(\Delta_{42}+\Delta_{42}^{(x)}\right)+\bar{L}_{2} \Delta_{42}^{(x)}+\left(\bar{L}_{1}-2 \ln x\right) \Delta_{42}+\right. \\
\left.\bar{L}_{3}\left(\bar{\theta}_{4}^{(x)}-\theta_{2}^{(x)}\right)+\bar{L}_{4}\left(\bar{\theta}_{4}-\theta_{2}\right)\right]
\end{gathered}
$$

where

$$
\begin{aligned}
& \bar{L}_{1}=\ln \left|\frac{\left(z-\rho_{2}^{2}\right)\left(\rho_{4}^{2}-z\right) x^{2}}{\left(x \rho_{4}^{2}-z\right)\left(x \rho_{2}^{2}-z\right)}\right|, \quad \bar{L}_{2}=\ln \left|\frac{\left(z-x^{2} \rho_{2}^{2}\right)\left(x^{2} \rho_{4}^{2}-z\right)}{x^{2}\left(x \rho_{4}^{2}-z\right)\left(x \rho_{2}^{2}-z\right)}\right|, \\
& \bar{L}_{3}=\ln \left|\frac{\left(z-x^{2} \rho_{2}^{2}\right)\left(x \rho_{4}^{2}-z\right)}{\left(x^{2} \rho_{4}^{2}-z\right)\left(x \rho_{2}^{2}-z\right)}\right|, \quad \bar{L}_{4}=\ln \left|\frac{\left(z-\rho_{2}^{2}\right)\left(x \rho_{4}^{2}-z\right)}{\left(\rho_{4}^{2}-z\right)\left(x \rho_{2}^{2}-z\right)}\right| .
\end{aligned}
$$

The $\Sigma_{H}$ is the sum of (23) and (25) divided by $4 \pi \alpha^{2} / Q_{1}^{2}$ :

$$
\begin{gathered}
\Sigma_{H}=\frac{\alpha}{2 \pi} \int_{1}^{\rho_{3}^{2}} \frac{d z}{z^{2}} \int_{x_{c}}^{1-\Delta} \frac{1+x^{2}}{1-x} d x\left[(L-1)\left(\Delta_{42}+\Delta_{42}^{(x)}\right)+\widetilde{K}\left(x, z ; \rho_{4}, \rho_{2}\right)\right], \\
\widetilde{K}=\frac{(1-x)^{2}}{1+x^{2}}\left(\Delta_{42}+\Delta_{42}^{(x)}\right)+\Delta_{42} \bar{L}_{1}+\Delta_{42}^{(x)} \bar{L}_{2}+\left(\bar{\theta}_{4}^{(x)}-\theta_{2}^{(x)}\right) \bar{L}_{3}+\left(\bar{\theta}_{4}-\theta_{2}\right) \bar{L}_{4} .
\end{gathered}
$$

As one can see the auxiliary parameter $\theta_{0}$ disappears in expressions for $\Sigma^{H}$ and $\Sigma_{H}$, and large logarithm acquires the right appearence. Thus, the separate investigation of contributions due to collinear and semi-collinear kinematics simplifies the calculations and gives also the dipper understanding of underlying physics. The experience of this approach is very important for the study of CES when it needs to describe events which belong to electron cluster (or positron one) in a different way as compared with events do not. 
The different parts in r.h.s. of Eq.(4) depend on auxiliary infrared paramerter $\Delta$ but the sum does not. It has the following form:

$$
\begin{aligned}
\Sigma_{1}= & \frac{\alpha}{2 \pi}\left\{\int_{1}^{\rho_{3}^{2}} \frac{d z}{z^{2}}\left[-\Delta_{42}+\int_{x_{c}}^{1}\left((L-1) P_{1}(x)\left(\Delta_{42}+\Delta_{42}^{(x)}\right)+\frac{1+x^{2}}{1-x} \widetilde{K}\right) d x\right]\right. \\
& \left.+\int_{\rho_{2}^{2}}^{\rho_{4}^{2}} \frac{d z}{z^{2}}\left[-1+\int_{x_{c}}^{1}\left((L-1) P_{1}(x)\left(1+\theta_{3}^{(x)}\right)+\frac{1+x^{2}}{1-x} K\right) d x\right]\right\},
\end{aligned}
$$

where

$$
P_{1}(x)=\frac{1+x^{2}}{1-x} \theta(1-x-\Delta)+\left(2 \ln \Delta+\frac{3}{2}\right) \delta(1-x), \quad \Delta \rightarrow 0 .
$$

In order to make the elimination of $\Delta$-dependence more transparent one can use the following relations:

$$
\begin{aligned}
& \int_{x_{c}}^{1} P_{1}(x) d x=-\int_{0}^{x_{c}} \frac{1+x^{2}}{1-x} d x, \int_{x_{c}}^{1} P_{1}(x) \bar{\theta}_{3}^{(x)} d x=\bar{\theta}_{3}^{\left(x_{c}\right)} \int_{x_{c}}^{\sqrt{z} / \rho_{3}} \frac{1+x^{2}}{1-x} d x \\
& \int_{x_{c}}^{1} P_{1}(x) \bar{\Delta}_{42}^{(x)} d x=\theta_{4} \bar{\theta}_{4}^{\left(x_{c}\right)} \int_{x_{c}}^{\sqrt{z} / \rho_{4}} \frac{1+x^{2}}{1-x} d x-\theta_{2} \bar{\theta}_{2}^{\left(x_{c}\right)} \int_{x_{c}}^{\sqrt{z} / \rho_{2}} \frac{1+x^{2}}{1-x} d x
\end{aligned}
$$

where $\bar{\Delta}_{42}^{(x)}=\Delta_{42}-\Delta_{42}^{(x)}$.

The r.h.s. of Eq.(28) is the full first order QED correction to born SABS cross section at LEP1 for IES with switched off vacuum polarization. The latter can be taken into account by insertion the quantity $\left[1-\Pi\left(-z Q_{1}^{2}\right)\right]^{-2}$ under sign of z-integration (for $\Pi$ see $[3]$ and references therein).

\section{Second order correction}

The second order corection contains the contributions due to double photons (real and vrtual) emission and pair production. As in symmetrical case one needs to distinguish between the situations when additional photons attach only one fermion line (one-side emission) and two fermion lines (opposite-side emission) in corresponding Feynman's diagrams.

\subsection{The contribution of pair production}

Consider at first the contribution of the process of electron-positron pair production $\Sigma^{\text {pair }}$ to the second order correction:

$$
\Sigma^{\text {pair }}=\Sigma^{e^{+} e^{-}}+\Sigma_{e^{+} e^{-}}
$$


In order to get rid of the writing some formulae which have the same structure for both symmetrical and wide-narrow angular acceptance I will often send the reader to work [11] in which the details of computation are given for symmetrical case.

The experience of Section 2 allows to write the expression for $\Sigma^{e^{+}} e^{-}$when created electronpositron pair press to electron momentum direction, using the result of [11] for $\Sigma^{e^{+} e^{-}}$suitable for wide--wide angular acceptance. It needs only to change z-integration limits; $\left(\rho^{2}, 1\right) \rightarrow\left(\rho_{4}^{2}, \rho_{2}^{2}\right)$ and substitute $\rho_{3}$ instead of $\rho$ everywhere under integral sign. The result may be written as follows:

$$
\begin{aligned}
& \Sigma^{e^{+} e^{-}}=\frac{\alpha^{2}}{4 \pi^{2}} \int_{\rho_{2}^{2}}^{\rho_{4}^{2}} \frac{d z}{z^{2}} L\left\{L\left(1+\frac{4}{3} \ln \left(1-x_{c}\right)-\frac{2}{3} \int_{x_{c}}^{1} \frac{d x}{1-x} \bar{\theta}_{3}^{(x)}\right)-\frac{17}{3}-\frac{8}{3} \zeta_{2}-\right. \\
& -\frac{40}{9} \ln \left(1-x_{c}\right)+\frac{8}{3} \ln ^{2}\left(1-x_{c}\right)+\int_{x_{c}}^{1} \frac{d x}{1-x} \bar{\theta}_{3}^{(x)}\left(\frac{20}{9}-\frac{8}{3} \ln (1-x)\right)+ \\
& \left.+\int_{x_{c}}^{1}\left[L \bar{R}(x)\left(1+\theta_{3}^{(x)}\right)+\theta_{3}^{(x)} C_{1}\left(x, z ; \rho_{3}\right)+C_{2}(x)+d_{2}\left(x, z ; \rho_{3}\right)\right] d x\right\} \\
& \bar{R}(x)=(1+x)\left(\ln x-\frac{1}{3}\right)+\frac{1-x}{6 x}\left(4+7 x+4 x^{2}\right), \\
& C_{1}\left(x, z ; \rho_{3}\right)=-\frac{113}{9}+\frac{142}{9} x-\frac{2}{3} x^{2}-\frac{4}{3 x}-\frac{4}{3}(1+x) \ln (1-x)+\frac{2\left(1+x^{2}\right)}{3(1-x)}\left[2 \ln \left|\frac{x^{2} \rho_{3}^{2}-z}{x \rho_{3}^{2}-z}\right|-\right. \\
& \left.-3 L_{i 2}(1-x)\right]+\left(8 x^{2}+3 x-9-\frac{8}{x}-\frac{7}{1-x}\right) \ln x+\frac{2\left(5 x^{2}-6\right)}{1-x} \ln ^{2} x+R(x) \ln \frac{\left(x^{2} \rho_{3}^{2}-z\right)^{2}}{\rho_{3}^{4}}, \\
& C_{2}(x)=-\frac{122}{9}+\frac{133}{9} x+\frac{4}{3} x^{2}+\frac{2}{3 x}-\frac{4}{3}(1+x) \ln (1-x)+\frac{2\left(1+x^{2}\right)}{(1-x)} L_{i 2}(1-x)+ \\
& +\frac{1}{3}\left(-8 x^{2}-32 x-20+\frac{8}{x}+\frac{13}{1-x}\right) \ln x+3(1+x) \ln ^{2} x, \quad R(x)=2 \bar{R}(x)+\frac{2}{3}(1+x), \\
& d_{2}\left(x, z ; \rho_{3}\right)=\frac{2\left(1+x^{2}\right)}{3(1-x)} \ln \left|\frac{\left(z-x^{2}\right)\left(\rho_{3}^{2}-z\right)(z-1)}{(z-x)^{2}\left(x^{2} \rho_{3}^{2}-z\right)}\right|++R(x) \ln \left|\frac{\left(z-x^{2}\right)\left(\rho_{3}^{2}-z\right)(z-1)}{x^{2} \rho_{3}^{2}-z}\right| \text {. }
\end{aligned}
$$

The r.h.s. of Eq.(31) does not contain infrared auxiliary parameter because it includes the contributions due to real and virtual pair production. The contribution of hard pair takes into account both, collinear and semi-collinear kinematics, and this ensures the next-to-leading accuracy.

If created elctron-positron pair is emitted along of the positron momentum direction the corresponding expression requires more modifications. The source of such modifications is the semicollinear kinematics as we saw in Section 2 for the single photon emission. 
The strightforward calculation shows that for contribution of the semi-collinear region $\vec{p}_{+} \| \vec{p}_{-}$ (I use here notation $\vec{p}_{ \pm}$for 3 - momentum of created positron (electron)) one has to write into formula (28) of [11]

$$
\begin{gathered}
\left(\Delta_{42}+\Delta_{42}^{(x)}\right) \ln \frac{z}{\lambda^{2}}+\Delta_{42} \ln \left|\frac{\left(z-\rho_{2}^{2}\right)\left(\rho_{4}^{2}-z\right)}{\left(z-x \rho_{2}^{2}\right)\left(x \rho_{4}^{2}-z\right)}\right|+\Delta_{42}^{(x)} \ln \left|\frac{\left(z-x^{2} \rho_{2}^{2}\right)\left(x^{2} \rho_{4}^{2}-z\right)}{x^{2}\left(z-x \rho_{2}^{2}\right)\left(x \rho_{4}^{2}-z\right)}\right|+ \\
\left(\bar{\theta}_{4}-\theta_{2}\right) \ln \left|\frac{\left(z-\rho_{2}^{2}\right)\left(x \rho_{4}^{2}-z\right)}{\left(z-x \rho_{2}^{2}\right)\left(\rho_{4}^{2}-z\right)}\right|+\left(\bar{\theta}_{4}^{(x)}-\theta_{2}^{(x)}\right) \ln \left|\frac{\left(z-x^{2} \rho_{2}^{2}\right)\left(x \rho_{4}^{2}-z\right)}{\left(z-x \rho_{2}^{2}\right)\left(z-x^{2} \rho_{4}^{2}\right)}\right|
\end{gathered}
$$

instead of expression in curle brackets and change the upper limit of z-integration: $\rho \rightarrow \rho_{3}$.

For the contribution of semi-collinear region $\vec{p}_{+} \| \vec{q}_{1}$ the correspnding expression is (see Eq.(33) in [11])

$$
\Delta_{42}\left(\ln \frac{z}{\lambda^{2}}+\ln \left|\frac{\left(z-\rho_{2}^{2}\right)\left(\rho_{4}^{2}-z\right)}{x_{2}^{2} \rho_{2}^{2} \rho_{4}^{2}}\right|\right)+\left(\bar{\theta}_{4}-\theta_{2}\right) \ln \left|\frac{\rho_{4}^{2}\left(z-\rho_{2}^{2}\right)}{\rho_{2}^{2}\left(z-\rho_{4}^{2}\right)}\right|
$$

and for semi-collinear region $\vec{p}_{-} \| \vec{p}_{1}$ (see Eq.(38) in [11])

$$
\Delta_{42}^{(x)}\left(\ln \frac{z}{\lambda^{2}}+\ln \left|\frac{\left(z-x^{2} \rho_{2}^{2}\right)\left(x^{2} \rho_{4}^{2}-z\right)}{x_{1}^{2} x^{4} \rho_{2}^{2} \rho_{4}^{2}}\right|\right)+\left(\bar{\theta}_{4}^{(x)}-\theta_{2}^{(x)}\right) \ln \left|\frac{\rho_{4}^{2}\left(z-x^{2} \rho_{2}^{2}\right)}{\rho_{2}^{2}\left(z-x^{2} \rho_{4}^{2}\right)}\right| .
$$

For the symmetrical wide-wide angular acceptance $\rho_{3}=\rho_{4}=\rho, \rho_{2}=1$, and

$$
\Delta_{42} \rightarrow \theta\left(\rho^{2}-z\right) \theta(z-1), \Delta_{42}^{(x)} \rightarrow \theta\left(x^{2} \rho^{2}-z\right), \bar{\theta}_{4}^{(x)} \rightarrow \theta\left(z-x^{2} \rho^{2}\right), \bar{\theta}_{4}, \theta_{2}, \theta_{2}^{(x)} \rightarrow 0,
$$

and (33), (34), (35) reduce to corresponding expressions derived in [11] .

The modification of the contributions due to virtual, real soft and hard collinear pair production includes the change of z-integral upper limit $: \rho \rightarrow \rho_{3}$ and trivial change of $\theta$-functions under integral sign, namely: $\theta\left(x^{2} \rho^{2}-z\right) \rightarrow \Delta_{42}^{(x)}, \quad 1 \rightarrow \Delta_{42}$. The sum of all contributions has the following form:

$$
\begin{gathered}
\Sigma_{e^{+} e^{-}}=\frac{\alpha^{2}}{4 \pi^{2}} \int_{1}^{\rho_{3}^{2}} \frac{d z}{z^{2}} L\left\{L\left[\Delta_{42}\left(1+\frac{4}{3} \ln \left(1-x_{c}\right)\right)-\frac{2}{3} \int_{x_{c}}^{1} \frac{d x}{1-x} \bar{\Delta}_{42}^{(x)}\right]+\Delta_{42}\left(-\frac{17}{3}-\frac{8}{3} \zeta_{2}-\right.\right. \\
\left.-\frac{40}{9} \ln \left(1-x_{c}\right)+\frac{8}{3} \ln ^{2}\left(1-x_{c}\right)\right)+\int_{x_{c}}^{1} \frac{d x}{1-x} \bar{\Delta}_{42}^{(x)}\left(\frac{20}{9}-\frac{8}{3} \ln (1-x)\right)+\int_{x_{c}}^{1}\left[L \bar{R}(x)\left(\Delta_{42}+\Delta_{42}^{(x)}\right)+\right. \\
+\Delta_{42}^{(x)} C_{1}\left(x, z ; \rho_{2}\right)+\Delta_{42}\left(C_{2}(x)+\bar{d}_{2}\left(x, z ; \rho_{2}\right)\right)+\left(\bar{\theta}_{4}^{(x)}-\theta_{4}^{(x)}\right)\left(\frac{2\left(1+x^{2}\right)}{3(1-x)} \ln \left|\frac{\left(x^{2} \rho_{2}^{2}-z\right)\left(x \rho_{4}^{2}-z\right)}{\left(x^{2} \rho_{4}^{2}-z\right)\left(x \rho_{2}^{2}-z\right)}\right|+\right. \\
\left.+R(x) \ln \left|\frac{\left(x^{2} \rho_{2}^{2}-z\right) \rho_{4}^{2}}{\left(x^{2} \rho_{4}^{2}-z\right) \rho_{2}^{2}}\right|\right)+\left(\bar{\theta}_{4}-\theta_{4}\right)\left(\frac{2\left(1+x^{2}\right)}{3(1-x)} \ln \left|\frac{\left(x \rho_{4}^{2}-z\right)\left(z-\rho_{2}^{2}\right) \mid}{\left(x \rho_{2}^{2}-z\right)\left(z-\rho_{4}^{2}\right)}\right|+\right.
\end{gathered}
$$




$$
\begin{gathered}
\left.\left.\left.+R(x) \ln \left|\frac{\left(\rho_{2}^{2}-z\right) \rho_{4}^{2}}{\left(\rho_{4}^{2}-z\right) \rho_{2}^{2}}\right|\right)\right]\right\} \\
\bar{d}_{2}\left(x, z ; \rho_{2}\right)=\frac{2\left(1+x^{2}\right)}{3(1-x)} \ln \frac{\left(z-\rho_{2}^{2}\right)^{2}}{\left(z-x \rho_{2}^{2}\right)^{2}}+2 R(x) \ln \frac{z-\rho_{2}^{2}}{\rho_{2}^{2}} .
\end{gathered}
$$

By the help of (36) one can verify that r.h.s. of Eq.(36) goes over in corresponding expression for symmetrical angular acceptance.

\subsection{The contribution of one-side double photon emission}

In this Section I give the analytical expressions for all contributions into the second order correction which appear due to one-side two photon (real and virtual) emission. The master formula which does not contain infrared auxiliary parameter $\Delta$ is written only for leading approximation, and next-to-leading contribution to it is given in Apendix A.

As before it needs to differ the radiation along electron and positron momentum directions

$$
\begin{gathered}
\Sigma_{2}=\Sigma^{\gamma \gamma}+\Sigma_{\gamma \gamma}, \quad \Sigma^{\gamma \gamma}=\Sigma^{(S+V)^{2}}+\Sigma^{(S+V) H}+\Sigma^{H H}, \\
\Sigma_{\gamma \gamma}=\Sigma_{(S+V)^{2}}+\Sigma_{(S+V) H}+\Sigma_{H H} .
\end{gathered}
$$

The contribution of virtual and real soft photon is the same for both the electron and the positron emission

$$
\begin{aligned}
\Sigma_{(S+V)^{2}}= & \Sigma^{(S+V)^{2}}=\frac{\alpha^{2}}{\pi^{2}} \int_{\rho_{2}^{2}}^{\rho_{4}^{2}} \frac{d z}{z^{2}} L\left[L\left(2 \ln ^{2} \Delta+3 \ln \Delta+\frac{9}{8}\right)-\right. \\
& \left.4 \ln ^{2} \Delta-7 \ln \Delta+3 \zeta_{3}-\frac{3}{2} \zeta_{2}-\frac{45}{16}\right] .
\end{aligned}
$$

Virtual and real soft photon correction to single hard photon emission already differs for photon moving along the electron momentum direction and the positron one. In the first case corresponding contribution may be derived by the help of result for symmetrical detector (see[10], formula (50)) using the substitutions $\left(\rho_{4}^{2}, \rho_{2}^{2}\right)$ instead of $\left(\rho^{2}, 1\right)$ for z-integration limits and $\rho_{3}$ instead of $\rho$ under integral sign. Therefore,

$$
\begin{gathered}
\Sigma^{(S+V) H}=\frac{\alpha^{2}}{2 \pi^{2}} \int_{\rho_{2}^{2}}^{\rho_{4}^{2}} \frac{d z}{z^{2}} L \int_{x_{c}}^{1-\Delta} \frac{1+x^{2}}{1-x} d x\left\{( 2 \operatorname { l n } \Delta - \operatorname { l n } x + \frac { 3 } { 2 } ) \left[K\left(x, z ; \rho_{3}, 1\right)+\right.\right. \\
\left.+(L-1)\left(1+\theta_{3}^{(x)}\right)\right]+\frac{1}{2} \ln ^{2} x-\frac{(1-x)^{2}}{2\left(1+x^{2}\right)}+\left(1+\theta_{3}^{(x)}\right)(-2+\ln x-2 \ln \Delta)+\bar{\theta}_{3}^{(x)}\left[\frac{1}{2} L \ln x+\right.
\end{gathered}
$$




$$
\left.\left.+2 \ln \Delta \ln x-\ln x \ln (1-x)-\ln ^{2} x-L_{i 2}(1-x)-\frac{x(1-x)+4 x \ln x}{2\left(1+x^{2}\right)}\right]\right\}
$$

In order to obtain the expression for $\Sigma_{(S+V) H}$ it needs to change in r.h.s. of Eq.(39):

i) limits of z-integration: $\left(\rho_{4}^{2}, \rho_{2}^{2}\right) \rightarrow\left(\rho_{3}^{2}, 1\right)$,

$$
\text { ii) } K\left(x, z: \rho_{3}, 1\right) \rightarrow \widetilde{K}\left(x, z: \rho_{4}, \rho_{2}\right) ; \theta_{3}^{(x)} \rightarrow \Delta_{42}^{(x)}, \bar{\theta}_{3}^{(x)} \rightarrow \bar{\Delta}_{42}^{(x)}, \quad 1 \rightarrow \Delta_{42}
$$

The contribution of two hard photons emitted along electron momentum directon may be obtained in the same way as $\Sigma^{(S+V) H}$, using the known result for symmetrical detectors (see [10], Eq.(54)), namely:

$$
\begin{gathered}
\Sigma^{H H}=\frac{\alpha^{2}}{4 \pi^{2}} \int_{\rho_{2}^{2}}^{\rho_{4}^{2}} \frac{d z}{z^{2}} L \int_{x_{c}}^{1-2 \Delta} d x \int_{\Delta}^{1-x-\Delta} d x_{1} \frac{I^{H H}}{x_{1}\left(1-x-x_{1}\right)\left(1-x_{1}\right)^{2}}, \\
\bar{A}=\gamma \beta\left(\frac{L}{2}+\ln \frac{\left(x^{2} \rho_{3}^{2}-z\right)^{2}}{x^{2}\left(x\left(1-x_{1}\right) \rho_{3}^{2}-z\right)^{2}}\right)+\zeta \ln \frac{\left(1-x_{1}\right)^{2}\left(1-x-x_{1}\right)}{x x_{1}}+\gamma_{A}, \\
\bar{B}=\gamma \beta\left(\frac{L}{2}+\ln \left|\frac{x^{2}(z-1)\left(\rho_{3}^{2}-z\right)\left(z-x^{2}\right)\left(z-\left(1-x_{1}\right)^{2}\right)^{2}\left(\rho_{3}^{2} x\left(1-x_{1}\right)-z\right)^{2}}{\left(\rho_{3}^{2}\left(1-x_{1}\right)^{2}-z\right)^{2}\left(z-\left(1-x_{1}\right)\right)^{2}\left(z-x\left(1-x_{1}\right)\right)^{2}\left(\rho_{3}^{2} x^{2}-z\right)}\right|\right)+ \\
\bar{C}=\gamma \beta\left(L+2 \ln \mid \frac{\left(1-x_{1}\right)^{2} x_{1}}{x\left(1-x-x_{1}\right)}+\delta_{B},\right. \\
\left.\frac{x\left(\rho_{3}^{2}\left(1-x_{1}\right)^{2}-z\right)^{2}}{\left(1-x_{1}\right)^{2}\left(\rho_{3}^{2} x\left(1-x_{1}\right)-z\right)\left(\rho_{3}^{2}\left(1-x_{1}\right)-z\right)} \mid\right)-2\left(1-x_{1}\right) \beta-2 x\left(1-x_{1}\right) \gamma,
\end{gathered}
$$

where

$$
\begin{gathered}
\gamma=1+\left(1-x_{1}\right)^{2}, \quad \beta=x^{2}+\left(1-x_{1}\right)^{2}, \quad \zeta=x^{2}+\left(1-x_{1}\right)^{4} \\
\gamma_{A}=x x_{1}\left(1-x-x_{1}\right)-x_{1}^{2}\left(1-x-x_{1}\right)^{2}-2\left(1-x_{1}\right) \beta, \quad \delta_{B}=x x_{1}\left(1-x-x_{1}\right)-x_{1}^{2}\left(1-x-x_{1}\right)^{2}-2 x\left(1-x_{1}\right) \gamma
\end{gathered}
$$

Unfortunately, it is impossible to give such simple prescription as (41) in order to obtain $\Sigma_{H H}$ from Eqs.(42) and (43). In the case of radiation two hard photons along the positron momentum direction an additional detailed consideration of semi-collinear kinematics is required. All essential moments of such consideration shown in Section 2, and reader can make all calculations by the help of formulae given in Appendix B of ref.[10]. Here I give final result

$$
\begin{gathered}
\Sigma_{H H}=\frac{\alpha^{2}}{4 \pi^{2}} \int_{1}^{\rho_{3}^{2}} \frac{d z}{z^{2}} L \int_{x_{c}}^{1-2 \Delta} d x \int_{\Delta}^{1-x-\Delta} d x_{1} \frac{I_{H H}}{x_{1}\left(1-x-x_{1}\right)\left(1-x_{1}\right)^{2}}, \\
I_{H H}=\widetilde{A} \Delta_{42}^{(x)}+\widetilde{C} \Delta_{42}^{\left(1-x_{1}\right)}+\widetilde{B} \Delta_{42}+\left(\bar{\theta}_{4}^{(x)}-\theta_{2}^{(x)}\right) a+\left(\bar{\theta}_{4}^{\left(1-x_{1}\right)}-\theta_{2}^{\left(1-x_{1}\right)}\right) c+\left(\bar{\theta}_{4}-\theta_{2}\right) b,
\end{gathered}
$$




$$
\begin{gathered}
a=\gamma \beta \ln \left|\frac{\left(\rho_{4}^{2} x\left(1-x_{1}\right)-z\right)\left(\rho_{2}^{2} x^{2}-z\right)}{\left(\rho_{2}^{2} x\left(1-x_{1}\right)-z\right)\left(\rho_{4}^{2} x^{2}-z\right)}\right|, \quad b=\gamma \beta \ln \left|\frac{\left(\rho_{4}^{2}\left(1-x_{1}\right)-z\right)\left(\rho_{2}^{2}-z\right)}{\left(\rho_{2}^{2}\left(1-x_{1}\right)-z\right)\left(\rho_{4}^{2}-z\right)}\right|, \\
c=\gamma \beta \ln \left|\frac{\left(\rho_{4}^{2} x\left(1-x_{1}\right)-z\right)\left(\rho_{2}^{2}\left(1-x_{1}\right)^{2}-z\right)^{2}\left(\rho_{4}^{2}\left(1-x_{1}\right)-z\right)}{\left(\rho_{2}^{2} x\left(1-x_{1}\right)-z\right)\left(\rho_{4}^{2}\left(1-x_{1}\right)^{2}-z\right)^{2}\left(\rho_{2}^{2}\left(1-x_{1}\right)-z\right)}\right| \\
\widetilde{A}=\gamma \beta\left(\frac{L}{2}+\ln \left|\frac{\left(\rho_{4}^{2} x^{2}-z\right)\left(\rho_{2}^{2} x^{2}-z\right)}{x^{2}\left(\rho_{4}^{2} x\left(1-x_{1}\right)-z\right)\left(\rho_{2}^{2} x\left(1-x_{1}\right)-z\right)}\right|\right)+\zeta \ln \frac{\left(1-x_{1}\right)^{2}\left(1-x-x_{1}\right)}{x x_{1}}+\gamma_{A}, \\
\widetilde{B}=\gamma \beta\left(\frac{L}{2}+\ln \left|\frac{x^{2}\left(\rho_{4}^{2}-z\right)\left(\rho_{2}^{2}-z\right)}{\left(\rho_{4}^{2}\left(1-x_{1}\right)-z\right)\left(\rho_{2}^{2}\left(1-x_{1}\right)-z\right)}\right|\right)+\zeta \ln \frac{\left(1-x_{1}\right)^{2} x_{1}}{x\left(1-x-x_{1}\right)}+\delta_{B}, \\
\widetilde{C}=\gamma \beta\left(L+\ln \left|\frac{x^{2}\left(\rho_{4}^{2}\left(1-x_{1}\right)^{2}-z\right)^{2}\left(\rho_{2}^{2}\left(1-x_{1}\right)^{2}-z\right)^{2}}{\left(1-x_{1}\right)^{4}\left(\rho_{4}^{2} x\left(1-x_{1}\right)-z\right)\left(\rho_{2}^{2} x\left(1-x_{1}\right)-z\right)\left(\rho_{4}^{2}\left(1-x_{1}\right)-z\right)\left(\rho_{2}^{2}\left(1-x_{1}\right)-z\right)}\right|\right) \\
-2\left(1-x_{1}\right)(\beta+x \gamma) .
\end{gathered}
$$

As one can see the separate contributions in r.h.s. of Eq.(38) depend on infrared auxiliary parameter $\Delta$ but $\Sigma^{\gamma \gamma}$ and $\Sigma_{\gamma \gamma}$ do not. In order to eliminate $\Delta$-dependence analytically it needs to apply a lot efforts. Below I give leading terms and for next-to-leading ones see Appendix A.

$$
\begin{gathered}
\Sigma^{\gamma \gamma L}=\frac{\alpha^{2}}{4 \pi^{2}} \int_{\rho_{2}^{2}}^{\rho_{4}^{2}} \frac{d z}{z^{2}} L^{2} \int_{x_{c}}^{1} d x\left[\frac{1}{2}\left(1+\theta_{3}^{(x)}\right) P_{2}(x)+\int_{x}^{1} \frac{d t}{t} P_{1}(t) P_{1}\left(\frac{x}{t}\right) \theta_{3}^{(t)}\right], \\
\Sigma_{\gamma \gamma}^{L}=\frac{\alpha^{2}}{4 \pi^{2}} \int_{1}^{\rho_{3}^{2}} \frac{d z}{z^{2}} L^{2} \int_{x_{c}}^{1} d x\left[\frac{1}{2}\left(\Delta_{42}+\Delta_{42}^{(x)}\right) P_{2}(x)+\int_{x}^{1} \frac{d t}{t} P_{1}(t) P_{1}\left(\frac{x}{t}\right) \Delta_{42}^{(t)}\right],
\end{gathered}
$$

where

$$
\begin{gathered}
P_{2}(x)=P_{1} \otimes P_{1}=\int_{x}^{1} \frac{d t}{t} P_{1}(t) P_{1}\left(\frac{x}{t}\right)=\lim _{\Delta \rightarrow 0}\left\{\left[\left(2 \ln \Delta+\frac{3}{2}\right)^{2}-4 \zeta_{2}\right] \delta(1-x)+\right. \\
\left.+2\left[\frac{1+x^{2}}{1-x}\left(2 \ln (1-x)-\ln x+\frac{3}{2}\right)+\frac{1}{2}(1+x) \ln x-1+x\right] \theta(1-x-\Delta)\right\}, \\
\int_{0}^{1} P_{2}(x) d x=0 .
\end{gathered}
$$

The expressions (45) and (46) are not convenient for numerical calculations. The suitable ones may be written as follows

$$
\begin{aligned}
\Sigma^{\gamma \gamma L} & =\frac{\alpha^{2}}{4 \pi^{2}}\left\{-2 \int_{\rho_{2}^{2}}^{\rho_{4}^{2}} \frac{d z}{z^{2}} L^{2} \int_{0}^{x_{c}} P_{2}(x) d x-\int_{m_{23}}^{\rho_{4}^{2}} \frac{d z}{z^{2}} L^{2} \int_{x_{c}}^{\sqrt{z} / \rho_{3}}\left[P_{1}(x) g\left(\frac{x_{c}}{x}\right)+\frac{1}{2} P_{2}(x)\right] d x\right\}, \\
\Sigma_{\gamma \gamma}^{L} & =\frac{\alpha^{2}}{4 \pi^{2}}\left\{-2 \int_{\rho_{2}^{2}}^{\rho_{4}^{2}} \frac{d z}{z^{2}} L^{2} \int_{0}^{x_{c}} P_{2}(x) d x-\int_{m_{14}}^{\rho_{4}^{2}} \frac{d z}{z^{2}} L^{2} \int_{x_{c}}^{\sqrt{z} / \rho_{4}}\left[P_{1}(x) g\left(\frac{x_{c}}{x}\right)+\frac{1}{2} P_{2}(x)\right] d x\right\}+
\end{aligned}
$$




$$
\left.+\int_{m_{12}}^{\rho_{2}^{2}} \frac{d z}{z^{2}} L^{2} \int_{x_{c}}^{\sqrt{z} / \rho_{2}}\left[P_{1}(x) g\left(\frac{x_{c}}{x}\right)+\frac{1}{2} P_{2}(x)\right] d x\right\}
$$

where

$$
\begin{gathered}
g(y)=y+\frac{y^{2}}{2}+2 \ln (1-y), m_{23}=\max \left(\rho_{2}^{2}, x_{c}^{2} \rho_{3}^{2}\right) \\
m_{14}=\max \left(1, x_{c}^{2} \rho_{4}^{2}\right), m_{12}=\max \left(1, x_{c}^{2} \rho_{2}^{2}\right) .
\end{gathered}
$$

The last two formulae can be derived by means the relations given in Appendix B. The integration relative $x$-variable in Eqs.(45) and (46) may be performed by the help of the following formulae

$$
\begin{gathered}
\int P_{2}(y) d y=F_{2}(x), \quad \int^{x} P_{1}(y) g\left(\frac{x_{c}}{y}\right) d y=F_{g}(x), \quad \int^{x} P_{1}(y) d y=-g(x), \quad x<1 \\
F_{2}(x)=-2 x-\frac{x^{2}}{4}+\left(x+\frac{x^{2}}{2}\right) \ln \frac{x^{3}}{(1-x)^{4}}+4 \ln (1-x) \ln \frac{x}{1-x}+4 L_{i 2}(x), \\
F_{g}(x)=-\frac{x_{c}^{2}}{2 x}+\left(2 x+x^{2}\right) \ln x+\left(x_{c}+\frac{x_{c}^{2}}{2}\right) \ln \frac{x}{(1-x)^{2}}+\left(2 x_{c}+\frac{x_{c}^{2}}{2}-2 x-\frac{x^{2}}{2}\right) \ln \left(x-x_{c}\right)+ \\
+4 L_{i 2}(x)+4 L_{i 2}\left(\frac{1-x}{1-x_{c}}\right), \quad x_{c}<x<1 .
\end{gathered}
$$

Therefore, the second order leading contribution to SABS cross section at LEP1 can be expressed through integral relative z-variable only.

It is useful to note also that for CES the leading contributions in all orders of perturbation theory take into account the emission of photons in initial state only. Thus, the corresponding correction due to one-side two photon (real and virtual) emission will be read in this case as follows:

$$
\begin{aligned}
& \Sigma_{C E S}^{\gamma \gamma L}=-\frac{1}{8}\left(\frac{\alpha}{\pi}\right)^{2} \int_{\rho_{2}^{2}}^{\rho_{4}^{2}} \frac{d z}{z^{2}} L^{2}\left\{F_{2}\left(x_{c}\right)+\left[F_{2}\left(\frac{\sqrt{z}}{\rho_{3}}\right)-F_{2}\left(x_{c}\right)\right] \bar{\theta}_{3}^{\left(x_{c}\right)}\right\} \\
& \Sigma_{\gamma \gamma C E S}^{L}=-\frac{1}{8}\left(\frac{\alpha}{\pi}\right)^{2}\left\{\int_{\rho_{2}^{2}}^{\rho_{4}^{2}} \frac{d z}{z^{2}} L^{2} F_{2}\left(x_{c}\right)+\int_{1}^{\rho_{4}^{2}} \frac{d z}{z^{2}} L^{2}\left[F_{2}\left(\frac{\sqrt{z}}{\rho_{4}}\right)-F_{2}\left(x_{c}\right)\right] \bar{\theta}_{4}^{\left(x_{c}\right)}-\right. \\
& \left.-\int_{1}^{\rho_{2}^{2}} \frac{d z}{z^{2}} L^{2}\left[F_{2}\left(\frac{\sqrt{z}}{\rho_{2}}\right)-F_{2}\left(x_{c}\right)\right] \bar{\theta}_{2}^{\left(x_{c}\right)}\right\}
\end{aligned}
$$

\subsection{Second order correction due to opposite-side photon emission}

In this Section I calculate analytically the expression for

$$
\Sigma_{\gamma}^{\gamma}=\Sigma_{S+V}^{S+V}+\Sigma_{S+V}^{H}+\Sigma_{H}^{S+V}+\Sigma_{H}^{H}
$$


The quantity $\Sigma_{\gamma}^{\gamma}$ does not depend on infrared auxiliary parameter $\Delta$ because it contains all contributions due to virtual, real soft and hard photon emission.

The first term in r.h.s. of Eq.(55) takes into account only "oposite-side" virtual and real soft photon corrections

$$
\Sigma_{S+V}^{S+V}=\frac{\alpha^{2}}{\pi^{2}} \int_{\rho_{2}^{2}}^{\rho_{4}^{2}} \frac{d z}{z^{2}} L\left[L\left(4 \ln ^{2} \Delta+6 \ln \Delta+\frac{9}{4}\right)-6-14 \ln \Delta-8 \ln ^{2} \Delta\right] .
$$

The contribution of one-loop virtual and real soft photon corrections to hard single photon emission may be written as follows

$$
\begin{gathered}
\Sigma_{S+V}^{H}=\frac{\alpha^{2}}{2 \pi^{2}} \int_{\rho_{2}^{2}}^{\rho_{4}^{2}} \frac{d z}{z^{2}}\left[2(L-1) \ln \Delta+\frac{3}{2} L-2\right] \int_{x_{c}}^{1-\Delta} \frac{1+x^{2}}{1-x}\left[\left(1+\theta_{3}^{(x)}\right)(L-1)+K\left(x, z ; \rho_{3}, 1\right)\right], \quad(57) \\
\Sigma_{H}^{S+V}=\frac{\alpha^{2}}{2 \pi^{2}} \int_{1}^{\rho_{3}^{2}} \frac{d z}{z^{2}}\left[2(L-1) \ln \Delta+\frac{3}{2} L-2\right] \int_{x_{c}}^{1-\Delta} \frac{1+x^{2}}{1-x}\left[\left(\Delta_{42}+\Delta_{42}^{(x)}\right)(L-1)+\widetilde{K}\left(x, z ; \rho_{4}, \rho_{2}\right)\right] d x .
\end{gathered}
$$

In order to find the contribution of two opposite-side hard photon emission into $\Sigma_{\gamma}^{\gamma}$ it is convenient to use the factorization theorem for differential cross-sections of two-jets processes in QCD [16]. It reads as:

$$
\begin{gathered}
\Sigma_{H}^{H}=\frac{\alpha^{2}}{4 \pi^{2}} \int_{0}^{\infty} \frac{d z}{z^{2}} \int_{x_{c}}^{1-\Delta} d x_{1} \int_{x_{c} / x_{1}}^{1-\Delta} d x_{2} \frac{1+x_{1}^{2}}{1-x_{1}} \frac{1+x_{2}^{2}}{1-x_{2}} \Phi\left(x_{1}, z, ; \rho_{3}, 1\right) \Phi\left(x_{2}, z ; \rho_{4}, \rho_{2}\right), \\
\Phi\left(x, z, ; \rho_{3}, 1\right)=\left(\Delta_{31}+\Delta_{31}^{(x)}\right)(L-1)+\frac{(1-x)^{2}}{1+x^{2}}\left(\Delta_{31}+\Delta_{31}^{(x)}\right)+\Delta_{31} L_{1}+\Delta_{31}^{(x)} L_{2}, \\
\left(\bar{\theta}_{3}^{(x)}-\theta_{1}^{(x)}\right) L_{3}+\left(\bar{\theta}_{3}-\theta_{1}\right) \ln \mid \frac{\left(x \rho_{3}^{2}-z\right)(z-1)}{(z-x)\left(\rho_{3}^{2}-z\right) \mid} \\
\Phi\left(x, z, ; \rho_{4}, \rho_{2}\right)=\left(\Delta_{42}+\Delta_{42}^{(x)}\right)(L-1)+\widetilde{K}\left(x, z ; \rho_{4}, \rho_{2}\right), \\
\Delta_{31}=\theta_{3}-\theta_{1}, \quad \Delta_{31}^{(x)}=\theta_{3}^{(x)}-\theta_{1}^{(x)}, \quad \theta_{1}=\theta(1-z), \quad \theta_{1}^{(x)}=\theta\left(x^{2}-z\right) .
\end{gathered}
$$

The $\Delta$-dependence of separate terms in r.h.s. of Eq.(55) can be eliminated analytically in the whole sum. The leading contribution is expressed in terms of electron structure functions as follows

$$
\Sigma_{\gamma}^{\gamma L}=\frac{\alpha^{2}}{4 \pi^{2}} \int_{0}^{\infty} \frac{d z}{z^{2}} L^{2} \int_{x_{c}}^{1} d x_{1} \int_{x_{c} / x_{1}}^{1} d x_{2} P_{1}\left(x_{1}\right) P_{1}\left(x_{2}\right)\left(\Delta_{31}+\Delta_{31}^{\left(x_{1}\right)}\right)\left(\Delta_{42}+\Delta_{42}^{\left(x_{2}\right)}\right) .
$$

The next-to-leading contribution to $\Sigma_{\gamma}^{\gamma}$ is given in Appendix A. 
The form of $\Sigma_{\gamma}^{\gamma}$ suitable for numerical counting may be written in terms of functions $F_{2}(x)$ and $F_{g}(x)$ in the same manner as it was done at the end of Subsection 3.2

$$
\begin{gathered}
\Sigma_{\gamma}^{\gamma L}=\frac{\alpha^{2}}{4 \pi^{2}}\left\{-\int_{\rho_{2}^{2}}^{\rho_{4}^{2}} \frac{d z}{z^{2}} L^{2}\left[4(1) F_{2}\left(x_{c}\right)+2(1)\left(F_{g}\left(\frac{\sqrt{z}}{\rho_{3}}\right)-F_{g}\left(x_{c}\right)\right) \bar{\theta}_{3}^{\left(x_{c}\right)}-\right.\right. \\
-\int_{1}^{\rho_{4}^{2}} \frac{d z}{z^{2}} L^{2} 2(1)\left(F_{g}\left(\frac{\sqrt{z}}{\rho_{4}}\right)-F_{g}\left(x_{c}\right)\right) \bar{\theta}_{4}^{\left(x_{c}\right)}+\int_{1}^{\rho_{2}^{2}} \frac{d z}{z^{2}} L^{2} 2(1)\left(F_{g}\left(\frac{\sqrt{z}}{\rho_{2}}\right)-F_{g}\left(x_{c}\right)\right) \bar{\theta}_{2}^{\left(x_{c}\right)}+ \\
+\int_{x_{c} \rho_{3} \rho_{4}}^{\rho_{4}^{2}} \frac{d z}{z^{2}} L^{2}\left[F_{g}\left(\frac{\sqrt{z}}{\rho_{4}}\right)-F_{g}\left(\frac{x_{c} \rho_{3}}{\sqrt{z}}\right)+g\left(\frac{\sqrt{z}}{\rho_{3}}\right)\left(g\left(\frac{\sqrt{z}}{\rho_{4}}\right)-g\left(\frac{x_{c} \rho_{3}}{\sqrt{z}}\right)\right)\right]+ \\
+\int_{x_{c} \rho_{2}}^{1} \frac{d z}{z^{2}} L^{2}\left[F_{g}(\sqrt{z})-F_{g}\left(\frac{x_{c} \rho_{2}}{\sqrt{z}}\right)+g\left(\frac{\sqrt{z}}{\rho_{2}}\right)\left(g(\sqrt{z})-g\left(\frac{x_{c} \rho_{2}}{\sqrt{z}}\right)\right)\right]- \\
-\int_{x_{c} \rho_{4}}^{1} \frac{d z}{z^{2}} L^{2}\left[F_{g}\left(\frac{\sqrt{z}}{\rho_{4}}\right)-F_{g}\left(\frac{x_{c}}{\sqrt{z}}\right)+g(\sqrt{z})\left(g\left(\frac{\sqrt{z}}{\rho_{4}}\right)-g\left(\frac{x_{c}}{\sqrt{z}}\right)\right)\right]- \\
\left.-\int_{x_{c} \rho_{3} \rho_{2}}^{\rho_{2}^{2}} \frac{d z}{z^{2}} L^{2}\left[F_{g}\left(\frac{\sqrt{z}}{\rho_{3}}\right)-F_{g}\left(\frac{x_{c} \rho_{2}}{\sqrt{z}}\right)+g\left(\frac{\sqrt{z}}{\rho_{2}}\right)\left(g\left(\frac{\sqrt{z}}{\rho_{3}}\right)-g\left(\frac{x_{c} \rho_{2}}{\sqrt{z}}\right)\right)\right]\right\} .
\end{gathered}
$$

In the r.h.s. of Eq.(63) the figures into brackets are suitable for CES, when only initial state radiation it needs to take into account.

\section{Third order correction}

Inside the required accuracy it needs to keep only leading contribution into the third order correction. The latter becomes more important than next-to leading one for LEP2 because of increase of the energy. In order to evalulate it one can use the iteration up to the third order of the master equation for the electron structure function [13]

$$
D\left(x, \alpha_{e f f}\right)=D^{N S}\left(x, \alpha_{e f f}\right)+D^{S}\left(x, \alpha_{e f f}\right) .
$$

The iterative form of non-singlet component of Eq.(64) reads

$$
\begin{gathered}
D^{N S}\left(x, \alpha_{e f f}\right)=\delta(1-x)+\sum_{k=1}^{\infty} \frac{1}{k !}\left(\frac{\alpha_{e f f}}{2 \pi}\right)^{k} P_{1}(x)^{\otimes k}, \\
\underbrace{P_{1}(x) \otimes \cdots \otimes P_{1}(x)}_{k}=P_{1}(x)^{\otimes k}, \quad P_{1}(x) \otimes P_{1}(x)=\int_{x}^{1} P_{1}(t) P_{1}\left(\frac{x}{t}\right) \frac{d t}{t} .
\end{gathered}
$$


Up to third order singlet component of Eq.(64) looks as follows [13]

$$
D^{S}\left(x, \alpha_{e f f}\right)=\frac{1}{2 !}\left(\frac{\alpha_{e f f}}{2 \pi}\right)^{2} R(x)+\frac{1}{3 !}\left(\frac{\alpha_{e f f}}{2 \pi}\right)^{3}\left[2 P_{1} \otimes R(x)-\frac{2}{3} R(x)\right]
$$

where $\mathrm{R}(\mathrm{x})$ is defined by Eq.(31). Effective coupling $\alpha_{\text {eff }}$ in Eqs. (64) - (66) represents integral of running QED constant

$$
\frac{\alpha_{e f f}}{2 \pi}=\int_{0}^{L} \frac{\alpha d t}{2 \pi(1-\alpha t / 3 \pi)}=\frac{3}{2} \ln \left(1-\frac{\alpha L}{3 \pi}\right)^{-1} .
$$

The nonsinglet structure function describes the photon emission and pair production without taking into account the identity of final fermions, while singlet one is responsible just on identity effects.

Up to third order the electron structure function has the following form

$$
\begin{gathered}
D(x, L)=\delta(1-x)+\frac{\alpha L}{2 \pi} P_{1}(x)+\frac{1}{2}\left(\frac{\alpha L}{2 \pi}\right)^{2}\left(P_{2}(x)+\frac{2}{3} P_{1}(x)+R(x)\right)+ \\
\frac{1}{3}\left(\frac{\alpha L}{2 \pi}\right)^{3}\left[\frac{1}{2} P_{3}(x)+P_{2}(x)+\frac{4}{9} P_{1}(x)+\frac{2}{3} R(x)+R^{p}(x)\right], \quad R^{p}(x)=P_{1} \oplus R(x) .
\end{gathered}
$$

For functions $P_{3}(x)$ and $R^{p}(x)$ see [6,13 MS].

The factorization form of the differential cross-section [16] leads to

$$
\begin{gathered}
\Sigma^{L}=\int_{0}^{\infty} \frac{d z}{z^{2}} \int_{x_{c}}^{1} d x_{1} \int_{x_{c} / x_{1}}^{1} d x_{2} C\left(x_{1}, L\right) C\left(x_{2}, L\right), \\
C\left(x_{1}, L\right)=\int_{x_{1}}^{1} \frac{d t}{t} D(t) D\left(\frac{x_{1}}{t}\right) \Delta_{31}^{(t)}, \quad C\left(x_{2}, L\right)=\int_{x_{2}}^{1} \frac{d t}{t} D(t) D\left(\frac{x_{2}}{t}\right) \Delta_{42}^{(t)} .
\end{gathered}
$$

The expansion of $C\left(x_{1}, L\right)$ reads

$$
\begin{gathered}
C\left(x_{1}, L\right)=\delta\left(1-x_{1}\right) \Delta_{31}^{\left(x_{1}\right)}+\frac{\alpha L}{2 \pi} P_{1}\left(x_{1}\right)\left(\Delta_{31}^{\left(x_{1}\right)}+\Delta_{31}\right)+ \\
+\left(\frac{\alpha L}{2 \pi}\right)^{2}\left[C_{2}\left(x_{1}\right)\left(\Delta_{31}^{\left(x_{1}\right)}+\Delta_{31}\right)+\int_{x_{1}}^{1} \frac{d t}{t} \Delta_{31}^{(t)} \bar{C}_{2}\left(x_{1}, t\right)\right]+ \\
+\left(\frac{\alpha L}{2 \pi}\right)^{3}\left[C_{3}\left(x_{1}\right)\left(\Delta_{31}^{\left(x_{1}\right)}+\Delta_{31}\right)+\int_{x_{1}}^{1} \frac{d t}{t} \Delta_{31}^{(t)} \bar{C}_{3}\left(x_{1}, t\right)\right], \\
C_{2}(x)=\frac{1}{2} P_{2}(x)+\frac{1}{3} P_{1}(x)+\frac{1}{2} R(x), \quad \bar{C}_{2}(x, t)=P_{1}(t) P_{1}\left(\frac{x}{t}\right), \\
C_{3}(x)=\frac{1}{6} P_{3}(x)+\frac{1}{3} P_{2}(x)+\frac{4}{27} P_{1}(x)+\frac{2}{9} R(x)+\frac{1}{3} R^{p}(x),
\end{gathered}
$$




$$
\bar{C}_{3}(x, t)=P_{1}(t) C_{2}\left(\frac{x}{t}\right)+C_{2}(t) P_{1}\left(\frac{x}{t}\right),
$$

and the same for $C\left(x_{2}, L\right)$ with the substitution $x_{2}$ instead of $x_{1}$ and $\Delta_{42}^{\left(x_{2}\right)}\left(\Delta_{42}\right)$ instead of $\Delta_{31}^{\left(x_{1}\right)}\left(\Delta_{31}\right)$.

Because of $\theta$-functions under integral sign one has to distinguish between

$$
\int_{x}^{1} \frac{d t}{t} A(t) B\left(\frac{x}{t}\right) \Delta_{31}^{(t)} \text { and } \int_{x}^{1} \frac{d t}{t} B(t) A\left(\frac{x}{t}\right) \Delta_{31}^{(t)} .
$$

In the case of CES one has to acount the initial-state radiation only. Therefore instead of (70) it needs to write

$$
C_{C E S}\left(x_{1}, L\right)=\Delta_{31}^{\left(x_{1}\right)}\left[\delta\left(1-x_{1}\right)+\frac{\alpha L}{2 \pi} P_{1}\left(x_{1}\right)+\left(\frac{\alpha L}{2 \pi}\right)^{2} C_{2}\left(x_{1}\right)+\left(\frac{\alpha L}{2 \pi}\right)^{3} C_{3}\left(x_{1}\right)\right],
$$

and analogous for $C\left(x_{2}, L\right)$.

The last step is to write third order contribution in r.h.s. of Eq.(69):

$$
\begin{gathered}
\Sigma_{3}^{L}=\left(\frac{\alpha}{2 \pi}\right)^{3} \int_{0}^{\infty} \frac{d z}{z^{2}} L^{3} \int_{x_{c}}^{1} d x\left(Z_{1}+\int_{x_{c} / x}^{1} d x_{1} Z_{2}\right), \\
Z_{1}=\left(2 \Delta_{42}+\Delta_{42}^{(x)} \Delta_{31}+\Delta_{31}^{(x)} \Delta_{42}\right) C_{3}(x)+\int_{x}^{1} \frac{d t}{t}\left(\Delta_{42}^{(t)} \Delta_{31}+\Delta_{31}^{(t)} \Delta_{42}\right) \bar{C}_{3}(x, t), \\
Z_{2}=\left[\left(\Delta_{31}+\Delta_{31}^{(x)}\right)\left(\Delta_{42}+\Delta_{42}^{\left(x_{1}\right)}\right)+\left(\Delta_{31}+\Delta_{31}^{\left(x_{1}\right)}\right)\left(\Delta_{42}+\Delta_{42}^{(x)}\right)\right] P_{1}(x) C_{2}\left(x_{1}\right)+ \\
+P_{1}(x) \int_{x_{1}}^{1}\left[\Delta_{31}^{(t)} \Delta_{42}+\Delta_{42}^{(t)} \Delta_{31}+\Delta_{31}^{(x)} \Delta_{42}^{(t)}+\Delta_{42}^{(x)} \Delta_{31}^{(t)}\right] \frac{d t}{t} \bar{C}_{2}\left(x_{1}, t\right) .
\end{gathered}
$$

When writing expressions for $Z_{1}$ and $Z_{2}$ it is taken into account that $\Delta_{31} \Delta_{42}=\Delta_{42}$. In the case of CES the expressions for $Z_{1}$ and $Z_{2}$ may be written as follows:

$$
Z_{1}=\left(\Delta_{42}^{(x)} \Delta_{31}+\Delta_{31}^{(x)} \Delta_{42}\right) C_{3}(x), \quad Z_{2}=\left(\Delta_{42}^{(x)} \Delta_{31}^{\left(x_{1}\right)}+\Delta_{42}^{\left(x_{1}\right)} \Delta_{31}^{(x)}\right) P_{1}(x) C_{2}\left(x_{1}\right)
$$

Using the relations given in Appendix B the r.h.s. of Eq.(73) may be represented in the form suitable for numerical calculations as double integral relative $z$ - and $x$-variables. It may be written as follows:

$$
\Sigma_{3}^{L}=\Sigma_{3}^{0}+\Sigma_{0}^{3}+\Sigma_{2}^{1}+\Sigma_{1}^{2}
$$

where upper (down) index shows the number of additional particles (real and virtual) emitted by the electron (the positron). The one-side emission contribute to the r.h.s. of Eq.(75) as

$$
\Sigma_{3}^{0}+\Sigma_{0}^{3}=\left(\frac{\alpha}{2 \pi}\right)^{3}\left\{\int _ { \rho _ { 2 } ^ { 2 } } ^ { \rho _ { 4 } ^ { 2 } } \frac { d z } { z ^ { 2 } } L ^ { 3 } \left[-2 \int_{0}^{x_{c}} F_{p}(x) d x+2 \int_{x_{c}}^{1} F_{r}(x) d x-\right.\right.
$$




$$
\begin{gathered}
\left.-\bar{\theta}_{3}^{\left(x_{c}\right)} \int_{x_{c}}^{\sqrt{z} / \rho_{3}} F_{p r}\left(x, x_{c}\right) d x\right]-\int_{1}^{\rho_{4}^{2}} \frac{d z}{z^{2}} L^{3} \bar{\theta}_{4}^{\left(x_{c}\right)} \int_{x_{c}}^{\sqrt{z} / \rho_{4}} F_{p r}\left(x, x_{c}\right) d x+ \\
\left.+\int_{1}^{\rho_{2}^{2}} \frac{d z}{z^{2}} L^{3} \bar{\theta}_{2}^{\left(x_{c}\right)} \int_{x_{c}}^{\sqrt{z} / \rho_{2}} F_{p r}\left(x, x_{c}\right) d x\right\},
\end{gathered}
$$

where

$$
\begin{gathered}
F_{p}(x)=\frac{4}{3} P_{3}(x)+\frac{4}{3} P_{2}(x)+\frac{8}{27} P_{1}(x), \quad F_{r}(x)=\frac{4}{9} R(x)+\frac{5}{3} R^{p}(x), \\
F_{p r}\left(x, x_{c}\right)=\frac{1}{6} P_{3}(x)+\frac{1}{2} P_{2}(x)\left[\frac{2}{3}+g\left(\frac{x_{c}}{x}\right)\right]+P_{1}(x)\left[\frac{4}{27}+\frac{1}{2} f\left(\frac{x_{c}}{x}\right)+\right. \\
\left.+\frac{2}{3} g\left(\frac{x_{c}}{x}\right)+\frac{1}{2} r\left(\frac{x_{c}}{x} ; 1\right)\right]+R(x)\left[\frac{2}{9}+\frac{1}{2} g\left(\frac{x_{c}}{x}\right)\right]+\frac{1}{3} R^{p}(x) \\
r(z, 1)=\int_{z}^{1} R(x) d x=-\frac{22}{9}+z+z^{2}+\frac{4}{9} z^{3}-\left(\frac{4}{3}+2 z+z^{2}\right) \ln z \\
f(z)=-F_{2}(z) .
\end{gathered}
$$

In the case of CES the corresponding contribution may be derived by insertion of functions $F_{p}^{c}, F_{r}^{c}$ and $F_{p r}^{c}$ into the r.h.s of Eq.(76) instead of functions $F_{p}, F_{r}$ and $F_{p r}$, respectively, where

$$
F_{p r}^{c}(x)=C_{3}(x), \quad F_{p}^{c}(x)=\frac{1}{6} P_{3}(x)+\frac{1}{3} P_{2}(x)+\frac{4}{27} P_{1}(x), F_{r}^{c}(x)=\frac{2}{9} R(x)+\frac{1}{3} R^{p}(x) .
$$

The contribution due to opposite-side emission to r.h.s. of Eq.(75) reads

$$
\begin{gathered}
\Sigma_{2}^{1}+\Sigma_{1}^{2}=\left(\frac{\alpha}{2 \pi}\right)^{3} \int_{\rho_{2}^{2}}^{\rho_{4}^{2}} \frac{d z}{z^{2}} L^{3}\left[\int_{0}^{x_{c}}\left(-8 P_{3}(x)-\frac{8}{3} P_{2}(x)\right) d x+\right. \\
\left.+4 \int_{x_{c}}^{1} R^{p}(x) d x-\bar{\theta}_{3}^{\left(x_{c}\right)} \int_{x_{c}}^{\sqrt{z} / \rho_{3}}\left(H\left(x, x_{c}\right)+2 g\left(\frac{x_{c}}{x}\right) h\left(x ; \sqrt{z} / \rho_{3}\right)\right) d x\right]- \\
-\int_{1}^{\rho_{4}^{2}} \frac{d z}{z^{2}} L^{3} \bar{\theta}_{4}^{\left(x_{c}\right)} \int_{x_{c}}^{\sqrt{z} / \rho_{4}}\left(H\left(x, x_{c}\right)+2 g\left(\frac{x_{c}}{x}\right) h\left(x ; \sqrt{z} / \rho_{4}\right)\right) d x+ \\
+\int_{1}^{\rho_{2}^{2}} \frac{d z}{z^{2}} L^{3} \bar{\theta}_{2}^{\left(x_{c}\right)} \int_{x_{c}}^{\sqrt{z} / \rho_{2}}\left(H\left(x, x_{c}\right)+2 g\left(\frac{x_{c}}{x}\right) h\left(x ; \sqrt{z} / \rho_{2}\right)\right) d x+ \\
+\int_{x_{c} \rho_{3} \rho_{4}}^{\rho_{4}^{2}} \frac{d z}{z^{2}} L^{3}\left[\int_{x_{c} \rho_{4} / \sqrt{z}}^{\sqrt{z} / \rho_{3}}\left(P_{1}(x) G\left(\frac{x_{c}}{x} ; \frac{\sqrt{z}}{\rho_{4}}\right)+g\left(\frac{x_{c}}{x} ; \frac{\sqrt{z}}{\rho_{4}}\right) h\left(x ; \frac{\sqrt{z}}{\rho_{3}}\right)\right) d x+\left(\rho_{3} \leftrightarrow \rho_{4}\right)\right]+ \\
+\int_{x_{c} \rho_{2}}^{1} \frac{d z}{z^{2}} L^{3}\left[\int_{x_{c} \rho_{2} / \sqrt{z}}^{\sqrt{z} / 1}\left(P_{1}(x) G\left(\frac{x_{c}}{x} ; \frac{\sqrt{z}}{\rho_{2}}\right)+g\left(\frac{x_{c}}{x} ; \frac{\sqrt{z}}{\rho_{2}}\right) h\left(x ; \frac{\sqrt{z}}{1}\right)\right) d x+\left(\rho_{2} \leftrightarrow 1\right)\right]-
\end{gathered}
$$




$$
\begin{aligned}
& -\int_{x_{c} \rho_{3} \rho_{2}}^{\rho_{2}^{2}} \frac{d z}{z^{2}} L^{3}\left[\int_{x_{c} \rho_{2} / \sqrt{z}}^{\sqrt{z} / \rho_{3}}\left(P_{1}(x) G\left(\frac{x_{c}}{x} ; \frac{\sqrt{z}}{\rho_{2}}\right)+g\left(\frac{x_{c}}{x} ; \frac{\sqrt{z}}{\rho_{2}}\right) h\left(x ; \frac{\sqrt{z}}{\rho_{3}}\right)\right) d x+\left(\rho_{3} \leftrightarrow \rho_{2}\right)\right]- \\
& -\int_{x_{c} \rho_{4}}^{1} \frac{d z}{z^{2}} L^{3}\left[\int_{x_{c} \rho_{4} / \sqrt{z}}^{\sqrt{z} / 1}\left(P_{1}(x) G\left(\frac{x_{c}}{x} ; \frac{\sqrt{z}}{\rho_{4}}\right)+g\left(\frac{x_{c}}{x} ; \frac{\sqrt{z}}{\rho_{4}}\right) h\left(x ; \frac{\sqrt{z}}{1}\right)\right) d x+\left(\rho_{4} \leftrightarrow 1\right)\right],
\end{aligned}
$$

where

$$
\begin{gathered}
g(a ; b)=g(a)-g(b), \quad G(a ; b)=G(a)-G(b), \quad G(z)=\frac{1}{2} f(z)+\frac{1}{3} g(z)+\frac{1}{2} r(z), \\
H\left(x, x_{c}\right)=P_{1}(x)\left[2 f\left(\frac{x_{c}}{x}\right)+\frac{4}{3} g\left(\frac{x_{c}}{x}\right)+r\left(\frac{x_{c}}{x} ; 1\right)\right]+g\left(\frac{x_{c}}{x}\right)\left[P_{2}(x)+R(x)\right] \\
h(x ; \sqrt{z} / \rho)=\int_{x}^{\sqrt{z} / \rho} \frac{d t}{t} P_{1}(t) P_{1}\left(\frac{x}{t}\right)= \\
\frac{1+x^{2}}{1-x}\left(\frac{3}{2}+2 \ln \frac{(\sqrt{z} / \rho-x)(1-x)}{(1-\sqrt{z} / \rho) x}\right)-1+x-\frac{\sqrt{z}}{\rho}+\frac{x \rho}{\sqrt{z}}-(1+x) \ln \frac{\sqrt{z}}{x \rho} .
\end{gathered}
$$

Note that substitutions inside square brackets concern both, limits of $x$-integration and expressions under $x$-integral sign.

In the case of CES the r.h.s. of Eq.(77) requires the following modifications: i) coefficient at $P_{3}(x)$ has to be reduced eight times, coefficients at $P_{2}(x)$ and $R^{p}(x)$-four times; ii) it needs to suppouse $h=0$ and to substitute $H^{c}\left(x, x_{c}\right)$ instead of $H\left(x, x_{c}\right)$, where

$$
\begin{gathered}
H^{c}\left(x, x_{c}\right)=P_{1}(x)\left[\frac{1}{2} f\left(\frac{x_{c}}{x}\right)+\frac{2}{3} g\left(\frac{x_{c}}{x}\right)+\frac{1}{2} r\left(\frac{x_{c}}{x} ; 1\right)\right]+\frac{1}{2} g\left(\frac{x_{c}}{x}\right)\left[P_{2}(x)+R(x)\right] . \\
5 \text { The numerical results }
\end{gathered}
$$

The numerical calculations carried out for the beam energy $\epsilon=46.15 \mathrm{GeV}$, and limited angles of circular detectors as given after Eq.(3). The Born cross-section

$$
\sigma_{B}=\frac{4 \pi \alpha^{2}}{Q_{1}^{2}} \int_{\rho_{2}^{2}}^{\rho_{4}^{2}} \frac{d z}{z^{2}}\left(1-\frac{z \theta_{1}^{2}}{2}\right)
$$

(in symmetrical wide-wide case the limits of integration are 1 and $\rho_{3}^{2}$ ) equals $175.922 \mathrm{nb}$ for ww angular acceptance and 139.971nb for nn and wn ones. Formula (78) takes into account the contributions of the scattered diagram as well as the interference of scattered and annihilation ones. The contribution of pure annihilation diagram is proportional to $\theta_{1}^{4}$ and is negligible even 
on the born level. Note, that one has to reduce twice the coefficient at $\theta_{1}^{2}$ under integral sign in the r.h.s. of Eq.(78) if he want restrict himself with the contribution of the scattered diagram only. When calculating the QED corrections to the cross-section (78) I systematically ignore the terms proportional $\theta_{1}^{2}$, which have the double logarithmic asymptotic behavior [17] and equal parametrically to $(\alpha|t|) \ln ^{2}(|t| / s) /(\pi s)$. The last value is about 0.1 per mille as compared with unit for LEP1 conditions.

The results of the numerical calculations of QED correction with the switched off vacuum polarization are shown in the Tables $\mathbf{1}-\mathbf{3}$. For comparsion we give also the corresponding numbers derived by the help of Monte Carlo program BLUMI based on the YFS exponentiation $[3]$.

As one can see from the Table1 there is an approximately constant difference on the level of 0.3 per mille between our analytical and MC results inside first order correction. Because BLUMI compute the first order correction exactly [18] it may be think that this distiguish is caused by omitted in the present calculation terms mentioned above.

\begin{tabular}{|ccccccccc|}
\hline \multicolumn{4}{c}{ first order correction } & \multicolumn{5}{c|}{ second order correction } \\
\hline$x_{c}$ & blumi ww & ww & nn & wn & blumi ww & ww & nn & wn \\
\hline 0.1 & 166.046 & 166.008 & 130.813 & 134.504 & 166.892 & 166.958 & 131.674 & 134.808 \\
0.3 & 164.740 & 164.702 & 129.797 & 133.416 & 165.374 & 165.447 & 130.524 & 133.583 \\
0.5 & 162.241 & 162.203 & 128.001 & 131.428 & 162.530 & 162.574 & 128.474 & 131.127 \\
0.7 & 155.431 & 155.390 & 122.922 & 125.809 & 155.668 & 155.597 & 123.206 & 125.225 \\
0.9 & 134.390 & 134.334 & 106.478 & 107.945 & 137.342 & 137.153 & 108.820 & 109.667 \\
\hline
\end{tabular}

Table1. The SABS cross-section (in $\mathbf{n b}$ ) with first and second order photonic correction

In the Table2 I give the absolute values of the second order correction to SABS cross-section taking into account both leading and next-to-leading contributions. The correction due to pair production is small in accordance with the results of the work [6]. The second order photonic correction is represented as a sum of leading contribution and next-to-leading one. As one can see the next-to-leading part is not negligible . 


\begin{tabular}{|rcrrccc|}
\hline \multicolumn{4}{c}{ pair production } & \multicolumn{3}{c|}{ two photon emission } \\
\hline$x_{c}$ & ww & $\mathbf{n n}$ & wn & ww & nn & wn \\
\hline 0.1 & 0.007 & -0.004 & 0.015 & $0.742+0.208$ & $0.679+0.182$ & $0.249+0.091$ \\
0.3 & -0.033 & -0.033 & -0.020 & $0.546+0.199$ & $0.556+0.171$ & $0.069+0.098$ \\
0.5 & -0.058 & -0.050 & -0.041 & $0.140+0.231$ & $0.291+0.182$ & $-0.314+0.134$ \\
0.7 & -0.090 & -0.074 & -0.069 & $-0.027+0.234$ & $0.117+0.187$ & $-0.571+0.170$ \\
0.9 & -0.142 & -0.115 & -0.115 & $2.961-0.142$ & $2.458-0.116$ & $1.822-0.090$ \\
\hline
\end{tabular}

Table2. The second order absolute correction to SABS cross-section (in $\mathbf{n b}$ )

In the Table3 the absolute value of the leading third order correction and SABS cross-section with all corrections obtained in this work are shown. The third order one takes into account three photon emission and pair production accompanied by single photon radiation. At large values of $x_{c}$ this correction is comparable with second order next-to-leading one. This effect will increase in the conditions of LEP2.

\begin{tabular}{|crrrccc|}
\hline \multicolumn{2}{|c}{ third order correction } & \multicolumn{4}{c|}{ SABS cross-section at LEP1 } \\
\hline$x_{c}$ & ww & nn & wn & ww & nn & wn \\
\hline 0.1 & -0.055 & -0.047 & -0.006 & 166.910 & 131.623 & 134.817 \\
0.3 & -0.065 & -0.053 & -0.018 & 165.349 & 10.438 & 133.545 \\
0.5 & -0.036 & -0.040 & 0.004 & 162.472 & 128.384 & 131.090 \\
0.7 & 0.089 & 0.058 & 0.124 & 155.596 & 123.190 & 125.310 \\
0.9 & 0.291 & 0.220 & 0.331 & 137.307 & 108.927 & 109.893 \\
\hline
\end{tabular}

Table3. Leading third order correction and SABS cross-section as obtained in this work

As concerns the second order correction it needs to have the analytical formulae based on exponentiated form of the electron structure function in order to be consequent in the comparison with the BLUMI results. On the other hand, the comparison of given here the second order photonic correction, which includes the leading and next-to-leading contributions, with the corresponding numbers for non-exponentiated BLUMI version [3] was done recently in [22], and the agreement is very impressive.

\section{Conclusion}

In this paper analytical calculation of QED correction to SABS cross section at LEP1 are given for the case of inclusive event selection and wide-narrow angular acceptance. These include leading and next-to-leading contributions in first and second orders of perturbation theory and 
leading one in the third order. The leading contributions in the case of calorimeter event selection are obtained too for any form of final electron and positron clusters. Results are represented in the form of manifold integrals with definite limits, and functions under integral sign have not any physical singularities. No problem arises with infrared divergence and double counting.

The selection of essential Feynman diagrams, utilization of natural for this problem Sudakov's variables, impact factor representation of differential cross section due to t-channel photon exchange as well as electron structure function method and investigation of underlying kinematics were very useful along of the whole this work. It needs to emphasize separately the simplifications connected with impact factor representation which allows to represent the differential cross sections of two-jets processes in QED by factorized form. The latter allows to use cut-off $\theta$ functions for the final electron and positron independently on the level of the differential cross-section. The calculation does not require to go to c.m.s. of underlying subprocess (as in [6]) and escapes corresponding complications.

At this point I want to comment the analytical calculation of leading contribution due to photon emission and pair production carried out in [6]. Authors of these articles used as the master formula for description QED corrections to the SABS cross-section due to initial-state radiation the representation valid for cross sections of Drell-Yan process [19], electron-positron annihilation into muons (or hadrons) [20] and large angle Bhabha scattering [21]. But inside this set the SABS process has a very particular feature caused by the existence of two different scales. The first one is the momentum transfer squared $t$, and just this scale defines the value of the crosssection. The second scale is full c.m.s. energy squared $s=4 \epsilon^{2}$, and the quantity $\theta^{2} \sim|t| / s<<1$ has status of a small correction.

The $t$-scale physics is very simple and defined by peripheral interaction of the electron and the positron due to one photon exchange, provided momentum transfer is pure perpendicular : $t=-\vec{q}^{2}$. The $s$-scale physics is more complicated. On the born level it exhibits as contribution of an annihilation diagram and beside this permits the energy and longitudinal momentum exchange for the contribution of scattering diagram. The first order QED correction for $s$-scale cross-section includes the contributions of box diagrams, large angle photon emission and up-down interference because both, the eikonal representation for the scattering amplitude and the factorization form of the differential cross-section, breaks down. In the second order large angle pair production and 
appear.

The structure function used in [6] controls $t$-scale cross-section only and has not any relation to $s$-scale one because physics of different scales evolute by its own laws.

On the other hand, only scattered diagram contributes in born cross-section used in [6]. But everytime when somebody neglects annihilation diagram as compared with scattering one he must automatically neglect $\theta^{2}$ as compared with unit everywhere including the born cross-section (see comments to Eq.(78)) and experimental cuts in order to be consequent. Taking into account these arguments the master formula in [6] must be necessary simplified by eliminating terms proportional $\xi \sim|t| / s<<1$ and $\xi^{2}$ in the numerator of Eq.(5) and in the cutoff restrictions. After this it becames adequate to one obtained in [10] and used in this work.

Numerical evaluations shows good agreement with Monte Carlo calculations inside first order but the achievement of an agreement for high order corrections will require an additional efforts, connected with writing the version, based on the exponentiated form of the electron structure function for present analytical calculation.

\section{Acknowlegement}

Author thanks E. Kuraev, L. Trentadue, S. Jadach, B.W.L. Ward, G. Montagna and B. Pietrzyk for fruitful discussions and critical remarks as well as A. Arbuzov and G. Gach for the help in the numerical calculation. This work supported by INTAS grant 93-1867.

\section{Appendix A}

Let us begin with the consideration of the next-to-leading second order $\Delta$-independent contribution due to one-side two photons emission. At first I will give analytical expression for symmetrical case, because it was not published up to now in relevant form. (I do not introduce special notation for next-to-leading contribution to $\Sigma$ keeping in mind that only such kind terms are considered along this Appendix)

$$
\begin{gathered}
\Sigma^{\gamma \gamma}=\Sigma_{\gamma \gamma}=\frac{1}{4}\left(\frac{\alpha}{\pi}\right)^{2} \int_{1}^{\rho^{2}} \frac{d z}{z^{2}} L Y, \\
Y=y+\int_{x_{c}}^{1} d x\left\{A+\int_{0}^{1-x} d x_{1}\left[\frac{1}{x_{1}} 4 \frac{1+x^{2}}{1-x}\left(\theta_{\rho}^{(x)} l_{1}+l_{2}\right)+\left(-1-\frac{1+x}{1-x_{1}}-\right.\right.\right. \\
\left.\left.-\frac{x}{\left(1-x_{1}\right)^{2}}\right)\left(l_{4}+\theta_{\rho}^{(x)} l_{3}+2 \theta_{\rho}^{\left(1-x_{1}\right)} l_{5}\right)+\frac{2(1+x)}{1-x_{1}} \theta_{\rho}^{\left(1-x_{1}\right)}\right]-
\end{gathered}
$$




$$
\begin{aligned}
& \left.-4 \frac{1+x^{2}}{1-x} \bar{\theta}_{\rho}^{(x)}\left[\int_{1-\sqrt{z} / \rho}^{1-x} d x_{1}\left(\frac{1}{x_{1}} l_{5}+\frac{2}{x_{2}} \ln \frac{x}{1-x_{1}}\right)+\int_{0}^{\sqrt{z} / \rho-x} \frac{d x_{1}}{x_{1}} l_{6}\right]\right\}, \\
& y=12 \zeta_{3}+10 \zeta_{2}-\frac{45}{4}-16 \ln ^{2}\left(1-x_{c}\right)-28 \ln \left(1-x_{c}\right), \\
& A=\left(1+\theta_{\rho}^{(x)}\right)\left[2(5+2 x)+4(x+3) \ln (1-x)+4 \frac{1+x^{2}}{1-x} \ln x\right]+ \\
& +2 \frac{1+x^{2}}{1-x}\left[\left(\frac{3}{2}-\ln x\right) K(x, z ; \rho, 1)-\frac{1}{2} \ln ^{2} x-\frac{(1-x)^{2}}{2\left(1+x^{2}\right)}+\right. \\
& \left.+2 \ln (1-x)\left(\theta_{\rho}^{(x)} \ln \left|\frac{x^{2} \rho^{2}-z}{x \rho^{2}-z}\right|+\ln \left|\frac{(z-1)\left(z-x^{2}\right)\left(\rho^{2}-z\right)}{(z-x)^{2}\left(x \rho^{2}-z\right)}\right|\right)\right]+ \\
& +\bar{\theta}_{\rho}^{(x)}\left[\frac{16}{1-x} \ln (1-x)+\frac{14}{1-x}-(1-x) \ln x+\right. \\
& +2 \frac{1+x^{2}}{1-x}\left(-\frac{3}{2} \ln ^{2} x+3 \ln x \ln (1-x)-L_{i 2}(1-x)-\frac{x(1-x)+4 x \ln x}{2\left(1+x^{2}\right)}+\right. \\
& \left.\left.+\frac{(1+x)^{2}}{1+x^{2}} \ln \left|\frac{(\sqrt{z}-x \rho)}{\rho-\sqrt{z}}\right|+2 \ln \left|\frac{\sqrt{z}-x \rho}{\rho}\right| \ln \left|\frac{x\left(x \rho^{2}-z\right)}{x^{2} \rho^{2}-z}\right|\right)\right], \\
& l_{1}=\ln \left|\frac{\left(x^{2} \rho^{2}-z\right)\left(x \rho^{2}-z\right)}{\left(x\left(1-x_{1}\right) \rho^{2}-z\right)\left(x\left(x+x_{1}\right) \rho^{2}-z\right)}\right|, \quad l_{3}=\ln \left|\frac{\left(1-x_{1}\right)^{2}\left(1-x-x_{1}\right)\left(x^{2} \rho^{2}-z\right)^{2}}{x^{3} x_{1}\left(x\left(1-x_{1}\right) \rho^{2}-z\right)^{2}}\right| \text {, } \\
& l_{2}=\ln \left|\frac{(z-x)^{2}\left(z-\left(1-x_{1}\right)^{2}\right)\left(z-\left(x+x_{1}\right)^{2}\right)}{\left(z-\left(1-x_{1}\right)\right)\left(z-x\left(1-x_{1}\right)\right)\left(\left(x+x_{1}\right)-z\right)\left(x\left(x+x_{1}\right)-z\right)}\right|+ \\
& \ln \left|\frac{\left(\left(1-x_{1}\right)^{2} \rho^{2}-z\right)\left(\left(x+x_{1}\right)^{2} \rho^{2}-z\right)\left(x \rho^{2}-z\right)}{\left(\left(x+x_{1}\right) \rho^{2}-z\right)\left(\left(1-x_{1}\right) \rho^{2}-z\right)\left(x^{2} \rho^{2}-z\right)}\right|, \\
& l_{4}=\ln \left|\frac{\left(1-x_{1}\right)^{2} x x_{1}(z-1)\left(z-x^{2}\right)\left(z-\left(1-x_{1}\right)^{2}\right)^{2}}{x_{2}\left(z-\left(1-x_{1}\right)\right)^{2}\left(z-x\left(1-x_{1}\right)\right)^{2}}\right|+\ln \left|\frac{\left(\rho^{2}-z\right)\left(x\left(1-x_{1}\right) \rho^{2}-z\right)^{2}}{\left(x^{2} \rho^{2}-z\right)\left(\left(1-x_{1}\right)^{2} \rho^{2}-z\right)^{2}}\right| \text {, } \\
& l_{5}=\ln \left|\frac{x\left(\left(1-x_{1}\right)^{2} \rho^{2}-z\right)^{2}}{\left(1-x_{1}\right)^{2}\left(x\left(1-x_{1}\right) \rho^{2}-z\right)\left(\left(1-x_{1}\right) \rho^{2}-z\right)^{2}}\right|, \\
& l_{6}=\ln \left|\frac{\left(x \rho^{2}-z\right)\left(\left(x+x_{1}\right)^{2} \rho^{2}-z\right)^{2}}{\left(x^{2} \rho^{2}-z\right)\left(x\left(x+x_{1}\right) \rho^{2}-z\right)\left(\left(x+x_{1}\right) \rho^{2}-z\right)}\right| \text {. }
\end{aligned}
$$

For wide-narrow angular acceptance it needs to consider only the case of the positron emission $\Sigma_{\gamma \gamma}$, because the corresponding expression for the electron emission $\Sigma^{\gamma \gamma}$ is just eq.(A1) with $\left(\rho_{4}^{2}, \rho_{2}^{2}\right)$ as the limits of $z$-integration and $\rho_{3}$ instead of $\rho$ under the integral sign.

The analytical expression for $\Sigma_{\gamma \gamma}$ has the following form:

$$
\begin{gathered}
\Sigma_{\gamma \gamma}=\frac{1}{4}\left(\frac{\alpha}{\pi}\right)^{2} \int_{1}^{\rho_{3}^{2}} \frac{d z}{z^{2}} L A_{N}^{W}, \\
A_{N}^{W}=y \Delta_{42}+\int_{x_{c}}^{1} d x\left\{\Delta_{42}[4(4+3 x)+6(x+3) \ln (1-x)+(x-1+\right.
\end{gathered}
$$




$$
\begin{aligned}
& \left.\left.+4 \frac{1+x^{2}}{1-x}\right) \ln x\right]+\Delta_{42}^{(x)}\left[(1-x)(3+\ln x)+2(x+3) \ln (1-x)+4 \frac{1+x^{2}}{1-x} \ln x\right]+\bar{\Delta}_{42}^{(x)} \frac{2}{1-x}(4+ \\
& \left.+(1+x)^{2}\right) \ln (1-x)+2 \frac{(1+x)^{2}}{1-x}\left(\theta_{4} \bar{\theta}_{4}^{(x)} \ln \left|\frac{\sqrt{z}-x \rho_{4}}{\rho_{4}-\sqrt{z}}\right|-\theta_{2} \bar{\theta}_{2}^{(x)} \ln \left|\frac{\sqrt{z}-x \rho_{2}}{\rho_{2}-\sqrt{z}}\right|\right)+ \\
& +\frac{1+x^{2}}{1-x} B+\int_{0}^{1-x} d x_{1}\left[2 \frac{1+x^{2}}{(1-x) x_{1}}\left(\Delta_{42}^{(x)} l_{1+}+\Delta_{42} l_{2+}+\left(\bar{\theta}_{4}^{(x)}-\theta_{2}^{(x)}\right) l_{1-}+\left(\bar{\theta}_{4}-\theta_{2}\right) l_{2-}\right)+\right. \\
& +\left(-1-\frac{1+x}{1-x_{1}}-\frac{x}{\left(1-x_{1}\right)^{2}}\right)\left(\Delta_{42}^{(x)}\left(\ln \frac{\left(1-x_{1}\right)^{2} x_{2}}{x^{3} x_{1}}+l_{3+}\right)+\Delta_{42}\left(\ln \frac{\left(1-x_{1}\right)^{2} x x_{1}}{x_{2}}+l_{4+}\right)+\right. \\
& +\Delta_{42}^{\left(1-x_{1}\right)}\left(2 \ln \frac{x}{\left(1-x_{1}\right)^{2}}+l_{5+}\right)+\left(\bar{\theta}_{4}^{(x)}-\theta_{2}^{(x)}\right) l_{3-}+\left(\bar{\theta}_{4}-\theta_{2}\right) l_{4-}+ \\
& \left.\left.+\left(\bar{\theta}_{4}^{\left(1-x_{1}\right)}-\theta_{2}^{\left(1-x_{1}\right)}\right) l_{5-}\right)+2 \frac{1+x}{1-x_{1}} \Delta_{42}^{\left(1-x_{1}\right)}\right]+2 \frac{1+x^{2}}{1-x} \theta_{4} \bar{\theta}_{4}^{(x)}\left[\int _ { 1 - \sqrt { z } / \rho _ { 4 } } ^ { 1 - x } d x _ { 1 } \left(\frac{1}{x_{1}} \bar{l}_{6}-\right.\right. \\
& \left.\left.-\frac{4}{x_{2}} \ln \frac{x}{1-x_{1}}\right)+\int_{0}^{\sqrt{z} / \rho_{4}-x} \frac{d x_{1}}{x_{1}} \bar{l}_{7}\right]+2 \frac{1+x^{2}}{1-x} \theta_{2} \bar{\theta}_{2}^{(x)}\left[\int _ { 1 - \sqrt { z } / \rho _ { 2 } } ^ { 1 - x } d x _ { 1 } \left(\frac{1}{x_{1}} \tilde{l}_{6}+\right.\right. \\
& \left.\left.\left.+\frac{4}{x_{2}} \ln \frac{x}{1-x_{1}}\right)+\int_{0}^{\sqrt{z} / \rho_{2}-x} \frac{d x_{1}}{x_{1}} \tilde{l}_{7}\right]\right\} \\
& B=\Delta_{42}\left(-2 \ln ^{2} x+2 \ln (1-x) \ln \left|\frac{x^{4}\left(z-\rho_{2}^{2}\right)^{2}\left(z-x^{2} \rho_{2}^{2}\right)\left(x^{2} \rho_{4}^{2}-z\right)\left(\rho_{4}^{2}-z\right)^{2}}{\left(z-x \rho_{2}^{2}\right)^{3}\left(x \rho_{4}^{2}-z\right)^{3}}\right|\right)+ \\
& +\Delta_{42}^{(x)}\left(\ln ^{2} x+2 \ln (1-x) \ln \left|\frac{\left(z-x^{2} \rho_{2}^{2}\right)\left(x^{2} \rho_{4}^{2}-z\right)}{x^{4}\left(z-x \rho_{2}^{2}\right)\left(x \rho_{4}^{2}-z\right)}\right|\right)+(3-2 \ln x) \widetilde{K}\left(x, z ; \rho_{4}, \rho_{2}\right)+ \\
& +\bar{\Delta}_{42}^{(x)}\left(7-2 \ln x \ln (1-x)-2 \ln ^{2} x-2 L_{i 2}(1-x)-\frac{x(1-x)+4 x \ln x}{1+x^{2}}\right)+ \\
& +2\left(\bar{\theta}_{4}-\theta_{2}\right) \ln (1-x) \ln \left|\frac{\left(x \rho_{4}^{2}-z\right)^{3}\left(z-\rho_{2}^{2}\right)^{2}\left(z-x^{2} \rho_{2}^{2}\right)}{\left(\rho_{4}^{2}-z\right)^{2}\left(x^{2} \rho_{4}^{2}-z\right)\left(z-x \rho_{2}^{2}\right)^{3}}\right|+ \\
& +2\left(\bar{\theta}_{4}^{(x)}-\theta_{2}^{(x)}\right) \ln (1-x) \ln \left|\frac{\left(z-x^{2} \rho_{2}^{2}\right)\left(x \rho_{4}^{2}-z\right)}{\left(x^{2} \rho_{4}^{2}-z\right)\left(x \rho_{2}^{2}-z\right)}\right|+ \\
& +4 \theta_{4} \bar{\theta}_{4}^{(x)} \ln \left|\frac{x \rho_{4}-\sqrt{z}}{\rho_{4}}\right| \ln \left|\frac{x\left(x \rho_{4}^{2}-z\right)}{x^{2} \rho_{4}^{2}-z}\right|+4 \theta_{2} \bar{\theta}_{2}^{(x)} \ln \left|\frac{\sqrt{z}-x \rho_{2}}{\rho_{2}}\right| \ln \left|\frac{z-x^{2} \rho_{2}^{2}}{x\left(z-x \rho_{2}^{2}\right)}\right| \\
& l_{1 \pm}=(1 \pm \hat{c}) \ln \left|\frac{\left(z-x^{2} \rho_{2}^{2}\right)\left(z-x \rho_{2}^{2}\right)}{\left(z-x\left(1-x_{1}\right) \rho_{2}^{2}\right)\left(z-x\left(x+x_{1}\right) \rho_{2}^{2}\right)}\right| \text {, } \\
& l_{2 \pm}=(1 \pm \hat{c})\left[\ln \left|\frac{\left(z-x \rho_{2}^{2}\right)^{3}\left(z-\left(1-x_{1}\right)^{2} \rho_{2}^{2}\right)^{2}\left(z-\left(x+x_{1}\right)^{2} \rho_{2}^{2}\right)^{2}}{\left(z-x^{2} \rho_{2}^{2}\right)\left(z-x\left(1-x_{1}\right) \rho_{2}^{2}\right)\left(z-x\left(x+x_{1}\right) \rho_{2}^{2}\right)\left(z-\left(1-x_{1}\right) \rho_{2}^{2}\right)^{2}\left(z-\left(x+x_{1}\right) \rho_{2}^{2}\right)^{2}}\right|\right] \text {, } \\
& l_{3 \pm}=(1 \pm \hat{c}) \ln \left|\frac{z-x^{2} \rho_{2}^{2}}{z-x\left(1-x_{1}\right) \rho_{2}^{2}}\right|, \quad l_{4 \pm}=(1 \pm \hat{c}) \ln \left|\frac{z-\rho_{2}^{2}}{z-\left(1-x_{1}\right) \rho_{2}^{2}}\right| \\
& l_{5 \pm}=(1 \pm \hat{c}) \ln \left|\frac{\left(z-\left(1-x_{1}\right)^{2} \rho_{2}^{2}\right)^{2}}{\left(z-x\left(1-x_{1}\right) \rho_{2}^{2}\right)\left(z-\left(1-x_{1}\right) \rho_{2}^{2}\right)}\right|
\end{aligned}
$$




$$
\begin{gathered}
\tilde{l}_{6}=\ln \left|\frac{x^{2}\left(z-\left(1-x_{1}\right)^{2} \rho_{2}^{2}\right)^{4}}{\left(1-x_{1}\right)^{4}\left(z-x\left(1-x_{1}\right) \rho_{2}^{2}\right)^{2}\left(z-\left(1-x_{1}\right) \rho_{2}^{2}\right)^{2}}\right|, \\
\tilde{l}_{7}=\ln \left|\frac{\left(z-x \rho_{2}^{2}\right)^{2}\left(z-\left(x+x_{1}\right)^{2} \rho_{2}^{2}\right)^{4}}{\left(z-x^{2} \rho_{2}^{2}\right)^{2}\left(z-x\left(x+x_{1}\right) \rho_{2}^{2}\right)^{2}\left(z-\left(x+x_{1}\right) \rho_{2}^{2}\right)^{2}}\right|, \quad \bar{l}_{6}=-\hat{c} \tilde{l}_{6}, \bar{l}_{7}=-\hat{c} \tilde{l}_{7},
\end{gathered}
$$

where $x_{2}=1-x-x_{1}$, and $\hat{c}$ is the operator of the substitution

$$
\hat{c} f\left(\rho_{2}\right)=f\left(\rho_{4}\right) .
$$

One can verify that in the symmetrical limit formula (A.2) coincides with (A.1) one.

For opposite-side emission the next-to-leading contribution to $\Sigma$ in the symmetrical case reads

$$
\begin{gathered}
\Sigma_{\gamma}^{\gamma}=\left(\frac{\alpha}{\pi}\right)^{2} L \int_{0}^{\infty} \frac{d z}{z^{2}} T \\
T=A \theta_{\rho} \bar{\theta}_{1}-\int_{x_{c}}^{1} d x\left[\frac{1+x^{2}}{2(1-x)} N(x, z ; \rho, 1)+\Xi(x)+\frac{\bar{\Xi}(x)}{1-x}\right] \\
\times \int_{x_{c} / x_{1}}^{1} d x_{1}\left[\left(1+x_{1}\right) \Xi\left(x_{1}\right)+\frac{2 \bar{\Xi}\left(x_{1}\right)}{1-x_{1}}\right],
\end{gathered}
$$

where

$$
\begin{aligned}
A & =-6-14 \ln \left(1-x_{c}\right)-8 \ln ^{2}\left(1-x_{c}\right)+\int_{x_{c}}^{1} d x\{7(1+x)+ \\
& +\frac{1+x^{2}}{2(1-x)}\left[3 K(x, z ; \rho, 1)+7 \bar{\theta}_{\rho}^{(x)}\right]+2 \ln \frac{x-x_{c}}{x}\left[(3+x)\left(1+\theta_{\rho}^{(x)}\right)+\right. \\
& \left.\left.+\frac{4}{1-x} \bar{\theta}_{\rho}^{(x)}+\frac{1+x^{2}}{1-x} N(x, z ; \rho, 1)\right]+\frac{8}{1-x} \ln \frac{x\left(1-x_{c}\right)}{x-x_{c}}\right\}
\end{aligned}
$$

We introduce the following reduced notation for $\theta$-functions:

$$
\Xi(x)=\theta_{\rho} \bar{\theta}_{1}+\theta_{\rho}^{(x)} \bar{\theta}_{1}^{(x)}, \quad \bar{\Xi}(x)=\theta_{\rho} \bar{\theta}_{\rho}^{(x)}-\theta_{1} \bar{\theta}_{1}^{(x)}
$$

- The quantity $K(x, z ; \rho, 1)$ entering into espression for $A$ is the $K$-factor for single photon emission, and the quantity $N(x, z ; \rho, 1)$ may be derived by the help of Eq.(10) in the following way:

$$
N(x, z ; \rho, 1)=\left.\left(\widetilde{K}\left(x, z ; \rho_{4}, \rho_{2}\right)-\frac{(1-x)^{2}}{1+x^{2}}\left(\Delta_{42}+\Delta_{42}^{(x)}\right)\right)\right|_{\rho_{4}=\rho, \rho_{2}=1} .
$$

Note that $N(1, z ; \rho, 1)=0$. 
In the wide-narrow angular acceptance the corresponding formula for $\Sigma_{\gamma}^{\gamma}$ may be written as follows:

$$
\Sigma_{\gamma}^{\gamma}=\frac{\alpha^{2}}{\pi^{2}} L \int_{0}^{\infty} \frac{d z}{z^{2}} T_{N}^{W},
$$

where

$$
\begin{aligned}
T_{N}^{W} & =\widetilde{A}-\frac{1}{2}\left\{\int_{x_{c}}^{1} d x\left[\frac{1+x^{2}}{2(1-x)} N\left(x, z ; \rho_{3}, 1\right)+\Xi_{31}(x)+\frac{1}{1-x} \bar{\Delta}_{31}^{(x)}\right]\right. \\
& \times \int_{x_{c} / x}^{1} d x_{1}\left[\left(1+x_{1}\right) \Xi_{42}(x)+\frac{2}{1-x_{1}} \bar{\Delta}_{42}^{(x)}\right]+ \\
& +\int_{x_{c}}^{1} d x\left[\frac{1+x^{2}}{2(1-x)} N\left(x, z ; \rho_{4}, \rho_{2}\right)+\Xi_{42}(x)+\frac{1}{1-x} \bar{\Delta}_{42}^{(x)}\right] \\
& \left.\times \int_{x_{c} / x}^{1} d x_{1}\left[\left(1+x_{1}\right) \Xi_{31}(x)+\frac{2}{1-x_{1}} \bar{\Delta}_{31}^{(x)}\right]\right\}
\end{aligned}
$$

where

$$
\begin{aligned}
\widetilde{A} & =\left(-6-14 \ln \left(1-x_{c}\right)-8 \ln ^{2}\left(1-x_{c}\right)\right) \Delta_{42}+ \\
& +\int_{x_{c}}^{1} d x\left\{\Delta_{42}\left[7(1+x)+\frac{8}{1-x} \ln \frac{x\left(1-x_{c}\right)}{x-x_{c}}\right]+\right. \\
& +\frac{1+x^{2}}{2(1-x)}\left[\frac{3}{2} \Delta_{42} \widetilde{K}\left(x, z ; \rho_{3}, 1\right)+\frac{3}{2} \Delta_{31} \widetilde{K}\left(x, z ; \rho_{4}, \rho_{2}\right)+\right. \\
& \left.+\frac{7}{2}\left(\Delta_{42} \bar{\Delta}_{31}^{(x)}+\Delta_{31} \bar{\Delta}_{42}^{(x)}\right)\right]+\ln \frac{x-x_{c}}{x}\left[(3+x)\left(\Delta_{31} \Xi_{42}(x)+\Delta_{42} \Xi_{31}(x)\right)+\right. \\
& +\frac{4}{1-x}\left(\bar{\Delta}_{42}^{(x)} \Delta_{31}+\bar{\Delta}_{31}^{(x)} \Delta_{42}\right)+\frac{1+x^{2}}{1-x}\left(\Delta_{42} N\left(x, z ; \rho_{3}, 1\right)+\right. \\
& \left.\left.\left.+\Delta_{31} N\left(x, z ; \rho_{4}, \rho_{2}\right)\right)\right]\right\},
\end{aligned}
$$

and

$$
\begin{gathered}
\Xi_{42}(x)=\theta_{4} \bar{\theta}_{2}+\theta_{4}^{(x)} \bar{\theta}_{2}^{(x)}=\Delta_{42}+\Delta_{42}^{(x)} \\
\Xi_{31}(x)=\Delta_{31}+\Delta_{31}^{(x)}, \quad \bar{\Delta}_{31}^{(x)}=\Delta_{31}-\Delta_{31}^{(x)} .
\end{gathered}
$$

It is obvious that in symmetrical limit formula (A.7) coinsides with (A.4) one. 


\section{Appendix B}

Here I give some relations which were used in the process of analytical calculations and at numerical computations. For the case of emission along the electron momentum direction they reads

$$
\begin{aligned}
\int_{\rho_{2}^{2}}^{\rho_{4}^{2}} d z \int_{x_{c}}^{1} d x \bar{\theta}_{3}^{(x)} & =\int_{\rho_{2}^{2}}^{\rho_{4}^{2}} d z \bar{\theta}_{3}^{\left(x_{c}\right)} \int_{x_{c}}^{\sqrt{z} / \rho_{3}} d x, \\
\int_{\rho_{2}^{2}}^{\rho_{4}^{2}} d z \int_{x_{c}}^{1} d x \int_{0}^{1-x} d x_{1} \bar{\theta}_{3}^{\left(1-x_{1}\right)} & =\int_{\rho_{2}^{2}}^{\rho_{4}^{2}} d z \bar{\theta}_{3}^{\left(x_{c}\right)} \int_{x_{c}}^{\sqrt{z} / \rho_{3}} d x \int_{1-\sqrt{z} / \rho_{3}}^{1-x} d x_{1} .
\end{aligned}
$$

For the case of the emission along the positron momentum direction:

$$
\begin{aligned}
& \int_{1}^{\rho_{3}^{2}} d z \int_{x_{c}}^{1} d x\left[\bar{\theta}_{4}^{(x)}-\theta_{2}^{(x)}\right]=\int_{1}^{\rho_{3}^{2}} d z \int_{x_{c}}^{1} d x\left[\bar{\theta}_{4}-\theta_{2}+\theta_{4} \bar{\theta}_{4}^{(x)}+\theta_{2} \bar{\theta}_{2}^{(x)}\right] \\
& =\int_{1}^{\rho_{3}^{2}} d z\left\{\left(\bar{\theta}_{4}-\theta_{2}\right) \int_{x_{c}}^{1} d x+\theta_{4} \bar{\theta}_{4}^{\left(x_{c}\right)} \int_{x_{c}}^{\sqrt{z} / \rho_{4}} d x+\theta_{2} \bar{\theta}_{2}^{\left(x_{c}\right)} \int_{x_{c}}^{\sqrt{z} / \rho_{2}} d x\right\}, \\
& \int_{1}^{\rho_{3}^{2}} d z \int_{x_{c}}^{1} d x \int_{0}^{1-x} d x_{1}\left[\bar{\theta}_{4}^{\left(1-x_{1}\right)}-\theta_{2}^{\left(1-x_{1}\right)}\right]=\int_{1}^{\rho_{3}^{2}} d z \int_{x_{c}}^{1} d x\left\{\left(\bar{\theta}_{4}-\theta_{2}\right)+\int_{0}^{\sqrt{z} / \rho_{2}} d x_{1}\right. \\
& +\bar{\theta}_{4}^{\left(x_{c}\right)} \theta_{4} \int_{x_{c}}^{\sqrt{z} / \rho_{4}} d x \int_{1-\sqrt{z} / \rho_{4}}^{1-x} d x_{1}+\bar{\theta}_{2}^{\left(x_{c}\right)} \theta_{2} \int_{x_{c}}^{1-\sqrt{z} / \rho_{2}} d x
\end{aligned}
$$

Some additional relations arise for the case of the opposite-side emission. Let us consider first the integration limits restrictions for the product of $\theta$-functions in the symmetrical case:

$$
\theta_{3} \bar{\theta}_{3}^{\left(x_{1}\right)} \bar{\theta}_{3}^{\left(x_{2}\right)}, \quad \theta_{1} \bar{\theta}_{3}^{\left(x_{1}\right)} \bar{\theta}_{1}^{\left(x_{2}\right)}, \quad \theta_{1} \bar{\theta}_{1}^{\left(x_{1}\right)} \bar{\theta}_{1}^{\left(x_{2}\right)}
$$

At first it needs to use the formulae (B.1) and get rid $\bar{\theta}_{i}^{\left(x_{2}\right)}$ using the following changes: i) $\bar{\theta}_{i}^{\left(x_{2}\right)} \rightarrow$ $\bar{\theta}_{i}^{\left(x_{c} / x_{1}\right)}$, ii) the upper limit of $x_{2}$ integration in the case of $\bar{\theta}_{3}^{\left(x_{2}\right)}$ has to be replaced by $\left(\sqrt{z} / \rho_{3}\right)$ and in the case of $\bar{\theta}_{1}^{\left(x_{2}\right)}$ by $\sqrt{z}$.

Thus, there are three regions defined by following curves in $\left(z, x_{1}\right)$ plane:

$$
\begin{aligned}
& \rho^{2}=z, \quad z=x_{1}^{2} \rho^{2}, \quad z=\frac{x_{c}^{2} \rho^{2}}{x_{1}^{2}} \\
& 1=z, \quad z=x_{1}^{2} \rho^{2}, \quad z=\frac{x_{1}}{x_{c}^{2}} \\
& 1=z, \quad z=x_{1}^{2}, \quad z=\frac{x_{1}^{2} \rho^{2}}{x_{c}^{2}} .
\end{aligned}
$$


It easy to see that the limits of integrations may be transformed as follows:

$$
\begin{aligned}
\int \theta_{3} \bar{\theta}_{3}^{\left(x_{1}\right)} \bar{\theta}_{3}^{\left(x_{2}\right)} \rightarrow \int_{x_{c} \rho^{2}}^{\rho^{2}} d z \int_{x_{c} \rho / \sqrt{z}}^{\sqrt{z} / \rho} d x_{1} \int_{x_{c} / x_{1}}^{\sqrt{z} / \rho} d x_{2} \\
\int \theta_{3} \bar{\theta}_{1}^{\left(x_{1}\right)} \bar{\theta}_{1}^{\left(x_{2}\right)} \rightarrow \int_{x_{c} \rho}^{1} d z \int_{x_{c} / \sqrt{z}}^{\sqrt{z} / \rho} d x_{1} \int_{x_{c} / x_{1}}^{\sqrt{z}} d x_{2},
\end{aligned}
$$

and for $\int \theta_{1} \bar{\theta}_{1}^{\left(x_{1}\right)} \bar{\theta}_{1}^{\left(x_{2}\right)}$ the formulae may be obtained from the above ones by putting $\rho=1$. For the wide-narrow angular acceptance the prescription is similar:

$$
\int \theta_{4} \bar{\theta}_{4}^{\left(x_{1}\right)} \bar{\theta}_{3}^{\left(x_{2}\right)} \rightarrow \int_{x_{c} \rho_{3}}^{\rho_{4}^{2}} d z \int_{x_{c} \rho_{3} / \sqrt{z}}^{\sqrt{z} / \rho_{4}} d x_{1} \int_{x_{c} / x_{1}}^{\sqrt{z} / \rho_{3}} d x_{2} .
$$

The another variants of restrictions in wide-narrow ansular acceptancee may be transformed as follows:

$$
\begin{aligned}
\int \theta_{1} \bar{\theta}_{2}^{\left(x_{1}\right)} \bar{\theta}_{1}^{\left(x_{2}\right)} \rightarrow \int_{x_{c} \rho_{2}}^{1} d z \int_{x_{c} \rho_{2} / \sqrt{z}}^{\sqrt{z}} d x_{1} \int_{x_{c} / x_{1}}^{\sqrt{z} / \rho_{2}} d x_{2}, \\
\int \theta_{1} \bar{\theta}_{4}^{\left(x_{1}\right)} \bar{\theta}_{1}^{\left(x_{2}\right)} \rightarrow \int_{x_{c} \rho_{4}}^{1} d z \int_{x_{c} \rho_{4} / \sqrt{z}}^{\sqrt{z} / \rho_{4}} d x_{1} \int_{x_{c} / x_{1}}^{\sqrt{z} / \rho_{2}} d x_{2}, \\
\int \theta_{2} \bar{\theta}_{2}^{\left(x_{1}\right)} \bar{\theta}_{3}^{\left(x_{2}\right)} \rightarrow \int_{x_{c} \rho_{2} \rho_{3}}^{\rho_{2}^{2}} d z \int_{x_{c} \rho_{3} / \sqrt{z}}^{\sqrt{z} / \rho_{3}} d x_{1} \int_{x_{c} / x_{1}}^{\sqrt{z}} d x_{2} .
\end{aligned}
$$

\section{References}

1. The LEP Collaboration: ALEPH, DELPI, L3 and OPAL and the LEP Electroweak Working Group, CERN-PPE/95; B. Pietrzyk, preprint LAPP-Exp-94.18. Invited talk at the Conference on "Radiative Corrections: Status and Outlook". Galtinburg, TN, USA, 1994; I.C. Brock et al., Preprint CERN-PPE/96-89’ CMU-HEP/96-04, 27 June 1996.

2. Neutrino Counting in Z Physics at LEP, G. Barbiellini et al., L. Trentadue (conv.); G. Altarelli, R. Kleiss and C. Verzegnassi eds. CERN Report 89-08.

3. Events Generator for Bhabha Scattering, H. Anlauf et al., Conveners: S. Jadach and O. Nicrosini. Yellow Report CERN 96-01, v.2 p.229 - 298.

4. S. Jadach, E. Richter-Was, B.F.L. Ward and Z. Was, Comput.Phys.Commun. 70 (1992) 305. 
5. G. Montagna et al., Comput.Phys.Commun. 76 (1993) 328; M. Cacciori, G. Montagna F. Piccinini, Comput.Phys.Commun. 90 (1995) 301, CERN-TH/95-169; G. Montagna et al., Nucl.Phys.B 401 (1993) 3.

6. S. Jadach, M. Skrzypek and B.F.L. Ward, Phys.Rev. D 47 (1993) 3733; S. Jadach, E. Richter-Was, B.F.L. Ward and Z. Was, Phys.Lett. B 260 (1991) 438.

7. S. Jadach, E. Richter-Was, B.F.L. Ward and Z. Was, Phys.Lett. B 353 (1995) 349, 362; S. Jadach, M. Melles, B.F.L. Ward and S.A. Yost, Phys.Lett.B 377 (1996) 168.

8. W. Beenakker, F.A. Berends and S.C. van der Marck, Nucl.Phys. B 355 (1991) 281; W. Beenakker and B. Pietrzyk, Phys.Lett. B 304 (1993) 366.

9. M. Gaffo, H. Czyz, E. Remiddi, Nuovo Cim. 105 A (1992) 271; Int.J.Mod.Phys. 4 (1993) 591; Phys.Lett. /bf B 327 (1994) 369; G. Montagna, O. Nicrosini and F. Piccinini, Preprint $\mathrm{FNT} / \mathrm{T}-96 / 8$.

10. A.B. Arbuzov et al., Yellow Report CERN 95-03, p.369; preprint CERN-TH/95-313, UPRF95-438 (to be published in Nucl.Phys.).

11. A.B. Arbuzov, E. Kuraev, N.P. Merenkov and L. Trentadue, JETP 81 (1995) 638; Preprint CERN-TH/95-241, JINR-E2-95-110.

12. D.R. Yennie, S.C. Frautchi and H. Suura, Ann.Phys. 13 (1961) 379.

13. L.N.Lipatov, Sov.J.Nucl.Phys. 20 (1974) 94; G. Altarelli and G. Parisi, Nucl.Phys. B 126 (1977) 298; M. Skrzypek, Acta Phys.Pol. B 23 (1992) 135.

14. N.P. Merenkov, Sov.J.Nucl.Phys. 48 (1988) 1073; 50 (1989) 469.

15. T.D. Lee and M. Nauenberg, Phys.Rev. B 133 (1964) 1549.

16. H. Cheng and T.T. Wu, Phys.Rev.Lett. 23 (1969) 670; V.G. Zima and N.P. Merenkov, Yad.Fis. 25 (1976) 998; V.N. Baier, V.S. Fadin, V. Khoze and E. Kuraev, Phys.Rep. 78 (1981) 294. 
17. V.G. Gorshkov, Uspechi Fiz. Nauk 110 (1973) 45; F.A. Berends et al., Nucl. Phys. B 57 (1973) 371; E.A. Kuraev and G.V. Meledin, Nucl. Phys.B 122 (1977) 3582.

18. S. Jadach and B.W.L. Ward, Phys. Rev. D 40 (1989) 3582.

19. S. Drell and T.M. Yan, Phys. Rev. Lett, 25 (1970) 316.

20. E.A. Kuraev and V.S. Fadin, Sov. J. Nucl. Phys. 41 (1985) 466.

21. W. Beenakker, F.A. Berends and S.C. van der Marck, Nucl. Phys. B 349 (1991) 323.

22. A.B. Arbuzov et al., Phys. Lett. B (in press). 